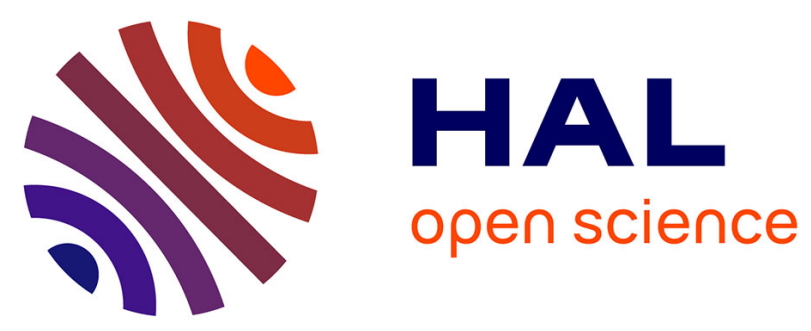

\title{
Comparison of theory and experiment for NAPL dissolution in porous media
}

\author{
T. Bahar, Fabrice Golfier, C. Oltean, E. Lefevre, C. Lorgeoux
}

\section{To cite this version:}

T. Bahar, Fabrice Golfier, C. Oltean, E. Lefevre, C. Lorgeoux. Comparison of theory and experiment for NAPL dissolution in porous media. Journal of Contaminant Hydrology, 2018, 211, pp.49-64. 10.1016/j.jconhyd.2018.03.004 . hal-02059287

\section{HAL Id: hal-02059287 \\ https://hal.univ-lorraine.fr/hal-02059287}

Submitted on 6 Mar 2019

HAL is a multi-disciplinary open access archive for the deposit and dissemination of scientific research documents, whether they are published or not. The documents may come from teaching and research institutions in France or abroad, or from public or private research centers.
L'archive ouverte pluridisciplinaire HAL, est destinée au dépôt et à la diffusion de documents scientifiques de niveau recherche, publiés ou non, émanant des établissements d'enseignement et de recherche français ou étrangers, des laboratoires publics ou privés. 


\title{
Comparison of theory and experiment for NAPL dissolution in porous media
}

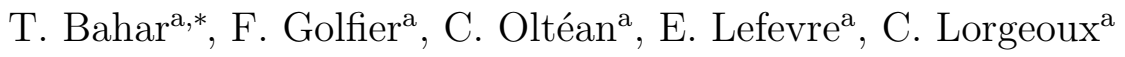 \\ ${ }^{a}$ Université de Lorraine, CNRS, CREGU, GeoRessources lab., Vandoeuvre-lès-Nancy Cedex, F-54518, \\ France
}

\begin{abstract}
Contamination of groundwater resources by an immiscible organic phase commonly called NAPL (Non Aqueous Phase Liquid) represents a major scientific challenge considering the residence time of such a pollutant. This contamination leads to the formation of NAPL blobs trapped in the soil and impact of this residual saturation cannot be ignored for correct predictions of the contaminant fate. In this paper, we present results of micromodel experiments on the dissolution of pure hydrocarbon phase (toluene). They were conducted for two values of the Péclet number. These experiments provide data for comparison and validation of a two-phase non-equilibrium theoretical model developped by Quintard and Whitaker (1994) using the volume averaging method. The model was directly upscaled from the averaged pore-scale mass balance equations. The effective properties of the macroscopic model were calculated over periodic unit cells designed from images of the experimental flow cell. Comparison of experimental and numerical results shows that the transport model predicts correctly - with no fitting parameters - the main mechanisms of NAPL mass transfer. The study highlights the crucial need of having a fair recovery of pore-scale characteristic lengths to predict the mass transfer coefficient with accuracy.
\end{abstract}

Keywords:

Porous media, NAPL dissolution, Upscaling, Volume averaging method

\section{List of symbols}

$A_{i j} \quad$ Interface between the $i$ - phase and $j$ - phase $(\mathrm{i}, \mathrm{j}: \omega, \beta, \gamma, k)$

$\mathbf{b}_{\beta}^{\prime} \quad$ Dimensionless form of the closure variable $\mathbf{b}_{\beta},(-)$

$C_{a}=\frac{\left\|\mathbf{v}_{\beta}\right\| \mu_{\beta}}{\sigma_{\beta \gamma}}$, Capillary number, $(-)$

$c_{A \beta} \quad$ Pore scale concentration of species $\mathrm{A}$ in the $\beta$ phase, $\left(\mathrm{kg} \mathrm{m} \mathrm{m}^{-3}\right)$

$\left\langle c_{A \beta}\right\rangle$ Averaged concentration of species $\mathrm{A}$ in the $\beta$ - phase, $\left(\mathrm{kg} \mathrm{m} \mathrm{m}^{-3}\right)$

$c_{A \beta}^{e q} \quad$ Concentration of species $\mathrm{A}$ in the $\beta$ - phase in equilibrium with $\rho_{\gamma},\left(\mathrm{kg} \mathrm{m} \mathrm{m}^{-3}\right)$

$c_{f} \quad$ Circularity, (-)

$c_{0} \quad$ Injected concentration in the complex $2 \mathrm{D}$ geometry, $\left(\mathrm{kg} \mathrm{m} \mathrm{m}^{-3}\right)$

\footnotetext{
*Corresponding author

Email address: tidjani.bahar@univ-lorraine.fr (T. Bahar)
} 
$D_{A \beta} \quad$ Diffusion coefficient of species A in the $\beta$ - phase, $\left(m^{2} s^{-1}\right)$

$\mathbf{D}_{\beta \omega}^{*} \quad$ Darcy scale effective dispersion tensor for the species A , $\left(m^{2} s^{-1}\right)$

$D_{f} \quad$ Feret diameter, $(\mathrm{cm})$

$\mathbf{d}_{\beta \omega}^{*} \quad$ A velocity-like effective transport coefficient, $\left(\mathrm{m} \mathrm{s}^{-1}\right)$

I Unit tensor

$K_{i j} \quad$ Equilibrium partitioning coefficient between the $i$-phase and $j$-phase for the species A, $(-)$

$L \quad$ Characteristic length associated with the averaging volume, $(m)$

$L_{c} \quad$ Characteristic length defining the macroscale, $(m)$

$l_{i} \quad$ Lattice vector for the $i$ th direction $(\mathrm{i}=1,2,3),(\mathrm{m})$

$l_{\beta} \quad$ Characteristic length associated to the $\beta$-phase, $(m)$

$\mathbf{n}_{i j} \quad$ Normal vector pointing outward from the $i$ - phase toward the $j$-phase

$p_{\beta} \quad$ Fluid pressure in the $\beta$-phase, $(P a)$

$P_{e}=\frac{\left\|\mathbf{v}_{\beta}\right\| l_{\beta}}{D_{A \beta}}$, Pore Péclet number, $(-)$

$R_{e}=\frac{\rho_{\beta}\left\|\mathbf{v}_{\beta}\right\| l_{\beta}}{\mu_{\beta}}$, Pore Reynolds number, (-)

$S_{c}=\frac{\mu_{\beta}}{\rho_{\beta} D_{A \beta}}$, Schmidt number, $(-)$

$S_{w i} \quad$ Initial saturation, $(-)$

$S h^{\prime}=\frac{\alpha_{A}^{*} l_{\beta}^{2}}{D_{A \beta}}$, Modified Sherwood number, $(-)$

$\mathbf{s}_{\beta}^{\prime} \quad$ Dimensionless form of the closure variable $s_{\beta},(-)$

$t$ Time, $(s)$

$t^{\prime} \quad$ Dimensionless time, $(-)$

$\mathbf{U}_{\beta \omega} \quad$ A velocity-like effective transport coefficient, $\left(\mathrm{m} \mathrm{s}^{-1}\right)$

$V \quad$ Averaging volume, $\left(\mathrm{m}^{3}\right)$

$V_{i} \quad$ Volume of the i-phase, $\left(m^{3}\right)$

$\mathbf{v}_{i} \quad$ Velocity of the $i$-phase, $\left(m s^{-1}\right)$

$\tilde{\mathbf{v}}_{i} \quad$ Spatial deviation of $\mathbf{v}_{i},\left(m s^{-1}\right)$

\section{Greek Symbols}

$\alpha_{A}^{*} \quad$ Mass-transfer coefficient for the species A, $\left(m s^{-1}\right)$

$\beta \quad$ Fluid phase

$\gamma \quad$ NAPL phase

$\epsilon \quad$ Total porosity

$\epsilon_{i} \quad$ Volume fraction of the $i$-phase

$\mu_{\beta} \quad$ Fluid dynamic viscosity, (Pa.s)

$\lambda_{f}$ Lacunarity, (-)

$\rho_{\gamma} \quad$ NAPL phase density, $\left(\mathrm{kg} \mathrm{m}^{-3}\right)$

$\rho_{\beta} \quad$ Water phase density, $\left(\mathrm{kg} \mathrm{m}^{-3}\right)$

$\sigma \quad$ Solid phase

$\sigma_{\beta \gamma} \quad$ water/toluene interfacial tension, $\left(N \mathrm{~m}^{-1}\right)$

$\langle.\rangle^{i}$ Intrinsic average for the i-phase 


\section{〈.〉 Superficial average}

\section{Introduction}

The problem of non-aqueous phase liquids (NAPLs) dissolution in porous media is often viewed in the context of groundwater pollution by hydrocarbon compounds (e.g., toluene, benzene, trichloroethylene). Among the sources of groundwater pollution, we can cite Abriola (1989): the drilling practices (i.e., drill-rig operations, transportation, illegal oil trapping) or hydrocarbon storage tanks (i.e., leaking underground storage tanks). Because of their high toxicity and low solubility in water and the associated risks to human health and environment, NAPLs represent a highly dangerous and durable contamination source of groundwater (Quintard and Whitaker, 1994; Schubert et al., 2007). Firstly, the pollutant migrates through the porous formation in the unsatured zone and then reaches the aquifer. Under the action of capillary forces, this contamination leads to the formation of NAPL blobs or ganglia trapped in the soil (Hunt et al., 1988). The assessment, design and implementation of efficient cleanup and remediation strategies for systems containing residual NAPL are of great importance for environmental and earth sciences research (National Research Council (NRC), 2005; Mainhagu and Brusseau, 2016; Atteia et al., 2017).

In the past years, various investigations were conducted to characterize the transport mechanisms of NAPLs in fluid saturated-porous media (i.e., morphology of NAPLs in porous media systems, dissolution processes, fingering phenomena). Historically, NAPL dissolution was first observed in laboratory column experiments (Miller and Poirier-Mcnell, 1990; Powers et al., 1992; Conrad et al., 1992; Powers et al., 1994; Yra et al., 2006; Javanbakht and Goual, 2016; Padgett et al., 2017). These works helped to give an insight on driving mechanisms but the obtained informations are averaged over the entire system since the breakthrough curves are measured at the column outlet. Considering the heterogeneous distribution of NAPL blobs in porous media, the effective properties calculated at the column-scale such as mass transfer coefficient or longitudinal dispersion cannot predict the impact of local heterogeneities neither describe the behavior of NAPL ganglia at the pore-scale. In addition, the phase distribution is not known. The measurement of microscale quantities such as interfacial areas are of critical importance to characterize the NAPL dissolution. Various three-dimensional imaging techniques (Culligan et al., 2006; Al-Raoush, 2009; Ghosh and Tick, 2013; Kashuk et al., 2014; Al-Raoush, 2014; Javanbakht et al., 2017) have been developed in the last decades to address this lack of data and characterize the microscale structure of NAPL ganglia. Recent improvements in the X-ray microtomography Armstrong et al., 2016, Garing et al., 2017) led to capture pore-scale interfacial processes and the NAPL dynamics but experimental constraints remain on the size of the sample domain, usually far from the size of the REV (Representative Elementary Volume). In parallel, experimental studies (Jia et al., 1999; Jeong et al., 2000; Corapcioglu et al., 2009a; Sahloul et al., 2002; Chomsurin and Werth, 2003; Armstrong and Berg, 2013; Kashuk et al., 2014) using transparent flow cells or micromodels have shown their ability to visualize the changes in NAPL saturation in porous media. Micromodels are 2D networks of transparent channels manufactured artificially and used to mimic natural porous media. Due to their design and 
properties including transparency, it is possible to visualize and extract data such as residual saturation and morphological characteristics of the NAPL blobs for a better assessment of mass transfer coefficient (Jeong et al., 2000; Corapcioglu et al., 2009a). Note that such investigations provide input data that serve as benchmarks to improve the understanding of the physics of NAPL entrapment and validate theoretical and numerical models.

Most of the proposed models (Corapcioglu and Baehr, 1987; Hunt et al., 1988; Quintard and Whitaker, 1994) that explore the NAPL blobs dissolution and mobilization in porous media are described in terms of local-equilibrium conditions or non-equilibrium conditions. The local mass equilibrium assumption, referring to conditions close to thermodynamic equilibrium, yields to define a single weighted-average concentration for the phases at equilibrium (see a discussion on this assumption in Seagren et al., 1999). On the contrary, the non-equilibrium assumption (or rate-limited assumption) better captures the complex dynamics of NAPL mass transfer but lead to a set of macroscopic mass balance equation per phase (Mobile et al., 2016). A first-order kinetics is classically used for describing mass transfer but more complex approaches can also be formulated (Wehrer et al., 2013). To simulate accurately the dissolution processes of NAPLs in porous media, however, both models require the knowledge of effective properties. The prediction of effective properties is difficult, particularly to describe the mass transfer processes between the water and NAPL phase. In the classical approach, modeling of mass transfer processes consist to use the phenomenological correlations (e.g., Gilland-Sherwood correlations) available in the literature (Miller and Poirier-Mcnell, 1990; Powers et al., 1992, 1994; Saba and Illangasekare, 2000). Nevertheless, these correlations do not take into account the impact of geometry and connectivity of the NAPL agglomerates as evidenced by several papers (Chomsurin and Werth, 2003; Zhao and Ioannidis, 2007; Kokkinaki et al., 2013; Al-Raoush, 2014; Mobile et al., 2016; Agaoglu et al., 2016). In practice, the proposed correlations may vary over several orders of magnitude following the structure of the studied porous medium. To solve this issue, a solution is to perform numerical simulations at the pore scale (e.g., using pore network models Zhao and Ioannidis (2003, 2007); Agaoglu et al. (2016)) to determine these effective properties and their evolution over time. This numerical upscaling, however, implies that the macro-scale balance equations and the related effective parameters are assumed to exist and to be known a priori that make their interpretation sometimes impossible and lead to errors in the development of averaged equations. Another alternative consists in using theoretical upscaling approaches (volume averaging, homogenization) Gray et al., 1993; Quintard and Whitaker, 1994) to determine and predict these effective properties based on the data at the pore scale. The explicit coupling between the micro-scale information and macroscopic coefficients is here maintained by the upscaling process. The difficulty, however, lies in the need to know at small scale the changes in geometry over time. The simplest solution is to suppose uniform dissolution of NAPL ganglia for example, but this remains a very reductive hypothesis. However, very few works have tried to compare these upscaling approaches with experimental results starting from a detailed knowledge of geometry evolution. An upscaled non-equilibrium model of NAPL dissolution based on the pore-scale averaged mass balance equations was proposed by Quintard and Whitaker (1994). Note that an extension of the model was proposed by Bahar et al. (2016) to consider bio-enhanced dissolution. In this 
model, the effective parameters (mass transfer coefficient and the effective dispersion) are determined by solving two closure problems on representative unit cells of the porous medium. Calculations of these properties on very simple unit cells were conducted by Ahmadi et al. (2001). Radilla et al. (1997, 1998) made an attempt to compare their column experiments with the predictions of the macroscopic model by considering simple geometry to calculate the effective coefficients but the lack of data on pore-scale structure made impossible a fair comparison.

In the present paper, we study both theoretically and experimentally residual NAPL aqueous dissolution and transport of dissolved species in a saturated 2D porous medium. The ultimate goal of this work is to provide a fair comparison between experimental results of dissolution and predictions of the upscaled model based on realistic unit cells designed from images of the experimental flow cell. The transport of dissolved species is modelled using a macroscopic model of multiphase transport at the Darcy-scale obtained from the volume averaging method (Quintard and Whitaker, 1994). The experimental set-up is made of a micromodel (i.e., a 2D transparent flow cell) used to study dissolution of toluene and provide data for comparison with the results of the theoretical model. In order to make this comparison, knowledge of the effective properties of the theoretical model is paramount. We assess the mass transfer coefficient and the effective dispersion by referring to the real architecture of the porous medium inferred from experimental data.

\section{Experimental study}

In this section, we will present the methodology that enabled the establishment of the experimental set-up. Then, we will focus on the presentation of results concerning the dissolution of NAPL blobs initially trapped at residual saturation.

\subsection{Experimental set-up and procedure}

A 2D transparent porous medium (micromodel) of dimensions $5 \mathrm{~cm} \mathrm{x} 5 \mathrm{~cm}$ and thickness $0.03 \mathrm{~cm}$ has been used for the experiment. The micromodel was made of etched glass by sandblasting. The pore network of micromodel was reconstructed from a tomographic image of sandstone in order to reproduce as faithfully as possible the natural porous media. The porosity and permeability of micromodel are $0.5 \pm 0.02$ and $3.73 \times 10^{-3} \mathrm{~cm} / \mathrm{s}$, respectively and the pore volume is $400 \mathrm{~mm}^{3}$ with an average pore size of $300 \mu \mathrm{m}$. Toluene $(>99.8 \%$ purity, Fisher Scientific Inc.), which is a common environnmental pollutant and the most water soluble of BTEX compounds, is used as a representative NAPL. Its physical properties are the following: fluid density $0.8669 \mathrm{~g} / \mathrm{mL}$, molecular weight $92.14 \mathrm{~g} / \mathrm{mol}$, molecular diffusion coefficient in water $8.6 \times 10^{-6} \mathrm{~cm}^{2} / \mathrm{s}$ and solubility in water $515 \mathrm{mg} / \mathrm{L}$. To trace the evolution of toluene within the micromodel we colored oil in red with Oil Red-O (Fisher Scientific) at a concentration of $0.5 \mathrm{~g} / \mathrm{L}$. A previous study (Jeong et al., 2000) has shown that this dye does not affect the physical and chemical properties of the solvent.

The experimental setup is illustrated in Fig. 1. The experimental apparatus consists of a horizontally-mounted micromodel and two syringe pumps (KD Scientific-Legato 110). These pumps ensure the injection of the toluene and water during the experiments. A 
camera (Canon EOS 400D) and the AZ100 Multizoom Microscope (Nikon Instruments Inc.) mounted directly on a system Charlyrobot Isel Automation controlled by the computer, were employed to follow spatial and temporal changes of NAPL blob within the porous medium. The experimental set-up is completed by gas chromatograph (Varian GC-450) to measure the concentration of toluene. First, the micromodel is saturated with deionized deaired water and then flooded with toluene until the irreducible water saturation is obtained. After this step, the micromodel is flushed with deionized deaired water at a flow rate of $5 \mathrm{ml} / \mathrm{h}$ until the residual oil saturation is reached. Then, the system is left at rest during twenty-four hours to get complete concentration equilibrium and a satisfactory initial residual saturation. At this point, NAPL is trapped in the form of isolated ganglia mainly driven by capillary forces, as suggested by the low value of the capillary number (cf. Table 1). This step is followed by the injection of demineralized water at a constant flow rate corresponding to the flow rate or Péclet number used for the dissolution experiments. Monitoring of dissolution is performed through two methods (Figure 11). First, we observe the changes in toluene saturation within the porous medium using CCD camera. The images of residual NAPL saturation are analyzed with ImageJ software. The area and perimeter of toluene blobs are directly measured with ImageJ from recorded two-dimensional images. In parallel, the dissolution of residual toluene is measured by gas chromatograph (GC) coupled with a flame iomization detector (FID). Due to the high volatility of toluene, headspace (HS) injection technique is used and peculiar precautions are taken for collecting samples and obtaining optimum reproducible results. The oven heat ramp was $10{ }^{\circ} \mathrm{C} / \mathrm{min}$ to $140{ }^{\circ} \mathrm{C}$ and the carrier gas was hydrogen at $1.8 \mathrm{~mL} / \mathrm{min}$ constant flow. At the flow cell outlet, a gas-tight syringe is used to extract $5 \mathrm{~mL}$ of solution into $10 \mathrm{~mL}$ sealed vials before HS-GC analysis. The toluene concentrations of samples are obtained by means of the calibration curve established using a standard protocol. 




Figure 1: Experimental set-up

\subsection{Experimental results}

\subsubsection{NAPL dissolution monitoring}

We investigate dissolution of trapped toluene at two different flow rates, respectively at $9.20 \times 10^{-3} \mathrm{~mL} / \mathrm{min}\left(P_{e}=7\right)$ and $2.81 \times 10^{-2} \mathrm{~mL} / \mathrm{min}\left(P_{e}=22\right)$. Note the above-mentioned Péclet number $\left(P_{e}\right)$ is a pore Péclet number defined as follows:

$$
P_{e}=\frac{\bar{v}_{\beta} l_{\beta}}{D_{A \beta}}
$$

where $\bar{v}_{\beta}$ is the average pore velocity, $l_{\beta}$ represents the pore characteristic length $(300 \mu \mathrm{m})$ and $D_{A \beta}$ is the molecular diffusion coefficient of toluene in water. The two-step experimental procedure of flow cell saturation and the injection protocol as described in the previous paragraph are the same for both experiments. The experimental data and the relevant dimensionless numbers are summarized in Table 1.

In both cases, we start with NAPL mainly trapped as large disconnected clusters. Due to the medium heterogeneity and the large aspect ratio of pores, NAPL trapping is preferentially found within the largest pores. This is consistent with by-passing mechanism where the wetting fluid (water) circumvents the largest pores through the smallest ones. However, isolated small ganglia due to snap-off can also be found, especially at high flow rates. 
Table 1: Experimental data

\begin{tabular}{lcc}
\hline Properties & Experiment I & Experiment II \\
\hline Flow rate $q(\mathrm{ml} / \mathrm{min})$ & $9.20 \times 10^{-3}$ & $2.81 \times 10^{-2}$ \\
\hline Pore-scale velocity $\bar{v}_{\beta}(\mathrm{cm} / \mathrm{min})$ & 0.12 & 0.37 \\
\hline Initial saturation $S_{w i}$ & 0.32 & 0.25 \\
\hline Pore Péclet number $\left(P_{e}\right)$ & 7 & 22 \\
\hline Pore Reynolds number $\left(R_{e}\right)$ & $6.13 \times 10^{-3}$ & $1.87 \times 10^{-2}$ \\
\hline Schmidt number $\left(S_{c}\right)$ & $1.16 \times 10^{3}$ & $1.16 \times 10^{3}$ \\
\hline Capillary number $\left(C_{a}\right)$ & $4.14 \times 10^{-7}$ & $1.28 \times 10^{-6}$ \\
\hline
\end{tabular}

Typical patterns observed during dissolution experiments are illustrated in Fig. 2. As soon as the co-current imbibition begins, we identify two mechanisms that contribute to NAPL recovery. First, NAPL remobilisation prevails. This phenomenon mainly occurs close to the inlet or near the highly polluted regions (areas marked in red on the images in Fig. 2) where high velocities - due to preferential flows - may be sufficient to overcome the forces of trapping, i.e. capillary forces). Anyway, this mechanism is present only at short times and never after $t=50 \mathrm{~h}$. Then, a progressive dissolution of toluene blobs over time is observed. Due to reduced water permeability in highly contaminated zones, preferential dissolution pathways occur (Fig. 2 - see the changes in regions marked in yellow). Similar results were obtained by Corapcioglu et al. (2009a). 


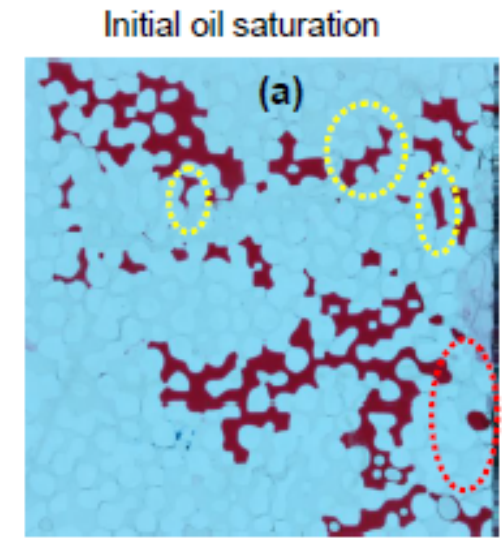

After $\mathrm{t}=50$ hours

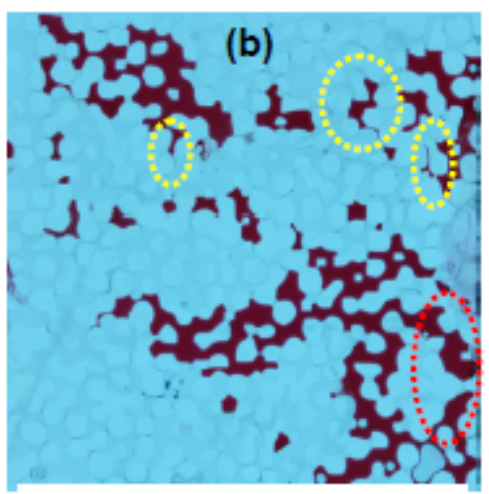

After $\mathrm{t}=100$ hours

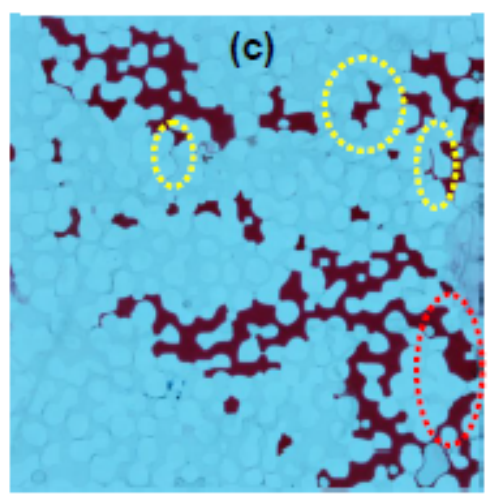

Experiment at $\mathrm{Pe}=7$

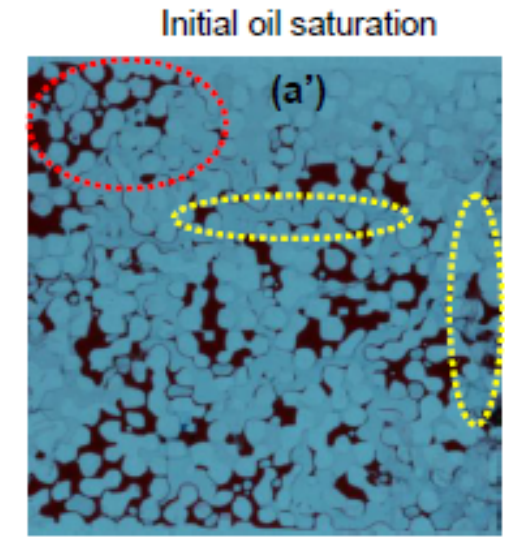

After $\mathrm{t}=50$ hours

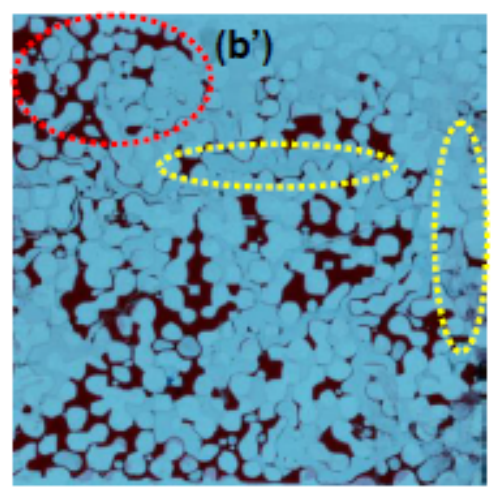

After $\mathrm{t}=100$ hours

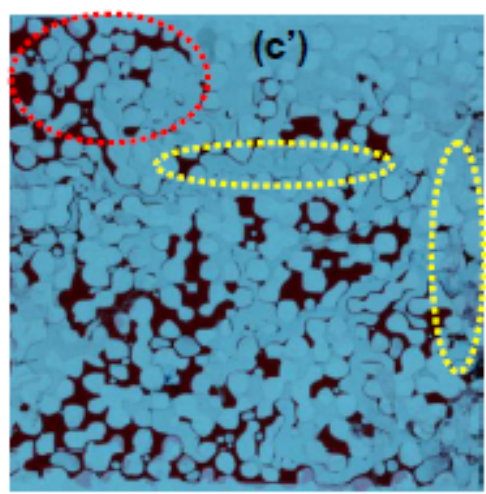

Experiment at $\mathrm{Pe}=22$

Figure 2: Progressive dissolution of toluene blobs in the micromodel for two experiments (images (a) and (a') corresponding to zero pore volumes injected, image (b) to 69 pore volumes injected, image (b') to 210 pore volumes injected, image (c) to 138 pore volumes injected and image (c') to 420 pore volumes injected). Direction of water flow was found from right to left

\subsubsection{Experimental uncertainty assessment}

First, experiments have been repeated twice to assess measurement uncertainty. Comparison of the resulting breakthrough curves are shown in Figs. 3 and 4 . We observe a relatively good repeatability of the experiments for the two values of Péclet. Discrepancy 
found at short time for the low Péclet experiment comes from remobilisation - inherently non-reproducible - of an isolated NAPL blob close to the outlet. The maximum relative error is on the order of $15 \%$ at high Péclet and $30 \%$ at low Péclet.

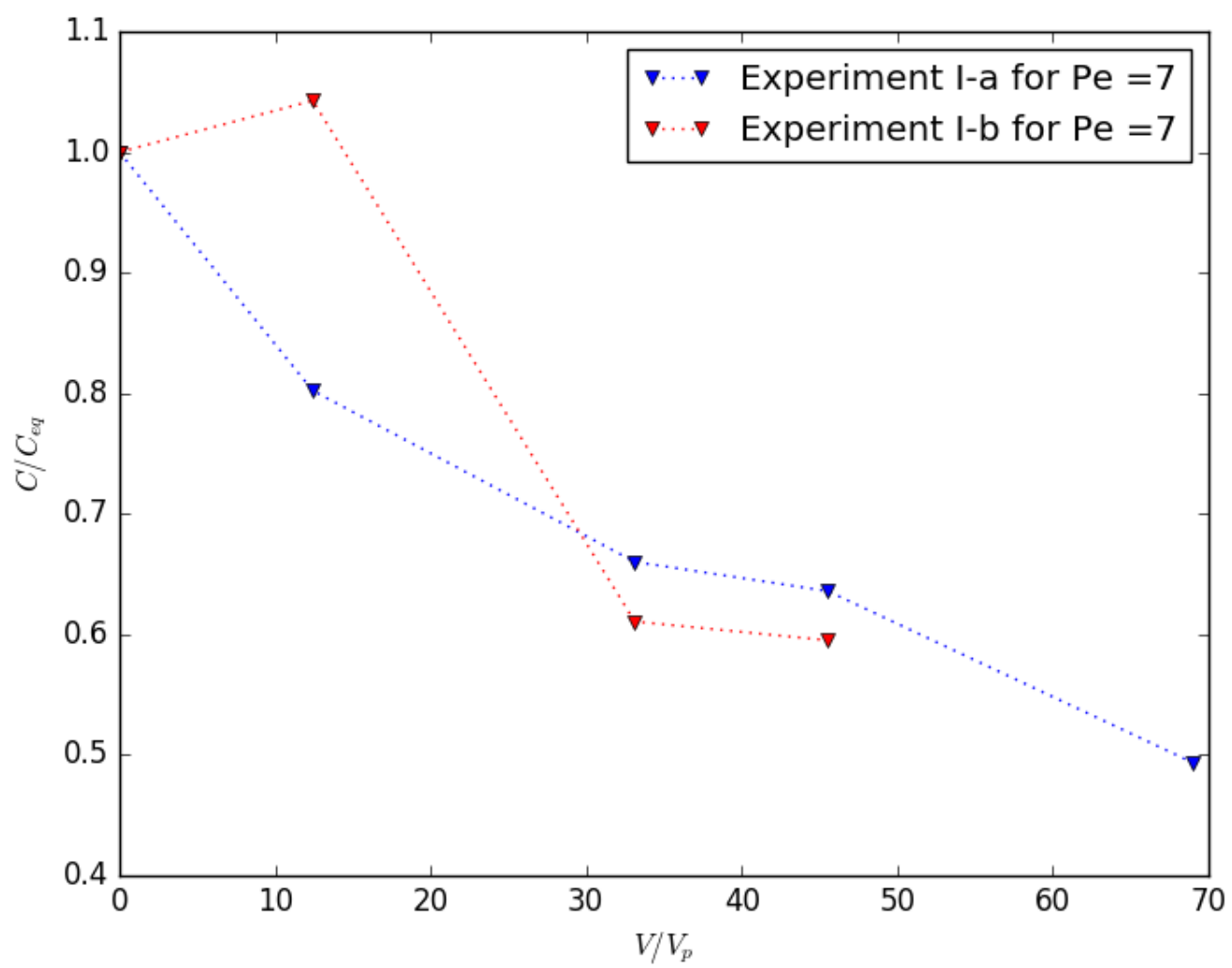

Figure 3: Toluene concentration at the outlet $\left(C / C_{e q}\right)$ versus pore volumes of water injected $\left(V / V_{p}\right)$ for $P_{e}=7$ 




Figure 4: Toluene concentration at the outlet $\left(C / C_{e q}\right)$ versus pore volumes of water injected $\left(V / V_{p}\right)$ for $P_{e}=22$

A second step has focused on the reliability of both measurement methods, i.e., camera monitoring and GC-analysis, from mass balance calculation on dissolved toluene. Mass balance analysis is carried out by comparison of the dissolved mass obtained by saturation measurement with the one determined from the breakthrough curve. Note that these both measures are not expected to be fully identical since the mass of dissolved toluene still present in the flow cell is not determined. This analysis helps to evaluate the accuracy of GC-concentration measurement (measurement uncertainty and sample recovery condition) and also to study the influence of the threshold used in image processing for evaluating residual saturation of toluene. Comparison is reported in Figs. 5 and 6 for the low- and high-Péclet experiments. Note that for the low-Péclet experiment, NAPL remobilisation and experimental troubles in sampling made a fair comparison of dissolved mass impossible at short times; mass balance comparison was thus limited to $V / V_{p}>150$ for $P_{e}=7$. Results indicate a broadly similar trend for both measures with nevertheless significant differences (up to $50 \%$ error on flux of dissolved toluene). This disagreement between results can be mainly explained by the phenomenon of remobilization previously reported (areas marked in red on the images in Fig. 2]. In fact, the remobilisation of the toluene blobs in the micro- 
model shown in Fig. 2 can lead to an overestimation of the residual saturation and hence of the solubilized mass. The experimental observations (Fig. 2) have identified this remobilisation of toluene blobs at short times and this occurence coincides strongly with the changes in the mass balance. The remobilized toluene blobs may subsequently become entrapped within the outlet tank of micromodel where they will continue to dissolve slowly, which justifies identical long-term concentrations for both methods. In addition, non-negligible measurement uncertainties remain in the oil saturation-estimation method by imaging analysis. Results at low Péclet indicate a low sensitivity of saturation measures to the value of thresholding but geometrical simplifications are made. Indeed, this calculation is based on the assumption that the micromodel is perfectly two-dimensional, which is not the case. The thickness of the micromodel may vary locally, thus modifying the measured volume. Moreover, the toluene droplets have a curvature according to the vertical. These various cumulative errors may lead to a significant uncertainty on the solubilized mass of toluene derived from imaging analysis because it depends directly on the geometric parameters of the pore network. As a conclusion, the measurement of output concentration seems the most reliable and this is the one we will use later for quantitative comparison with the numerical model. The monitoring of the saturation will be mainly used in a more qualitative manner, for the purpose of understanding the dissolution mechanisms involved and of identifying the most important geometrical features (e.g., pore size distribution, pore connectivity, NAPL surface area) required for the upscaling procedure as discussed in Section 4 . 




Figure 5: Mass balance of dissolved toluene as a function of the number of pore volumes injected $\left(V / V_{p}\right)$ for $P_{e}=7$ 


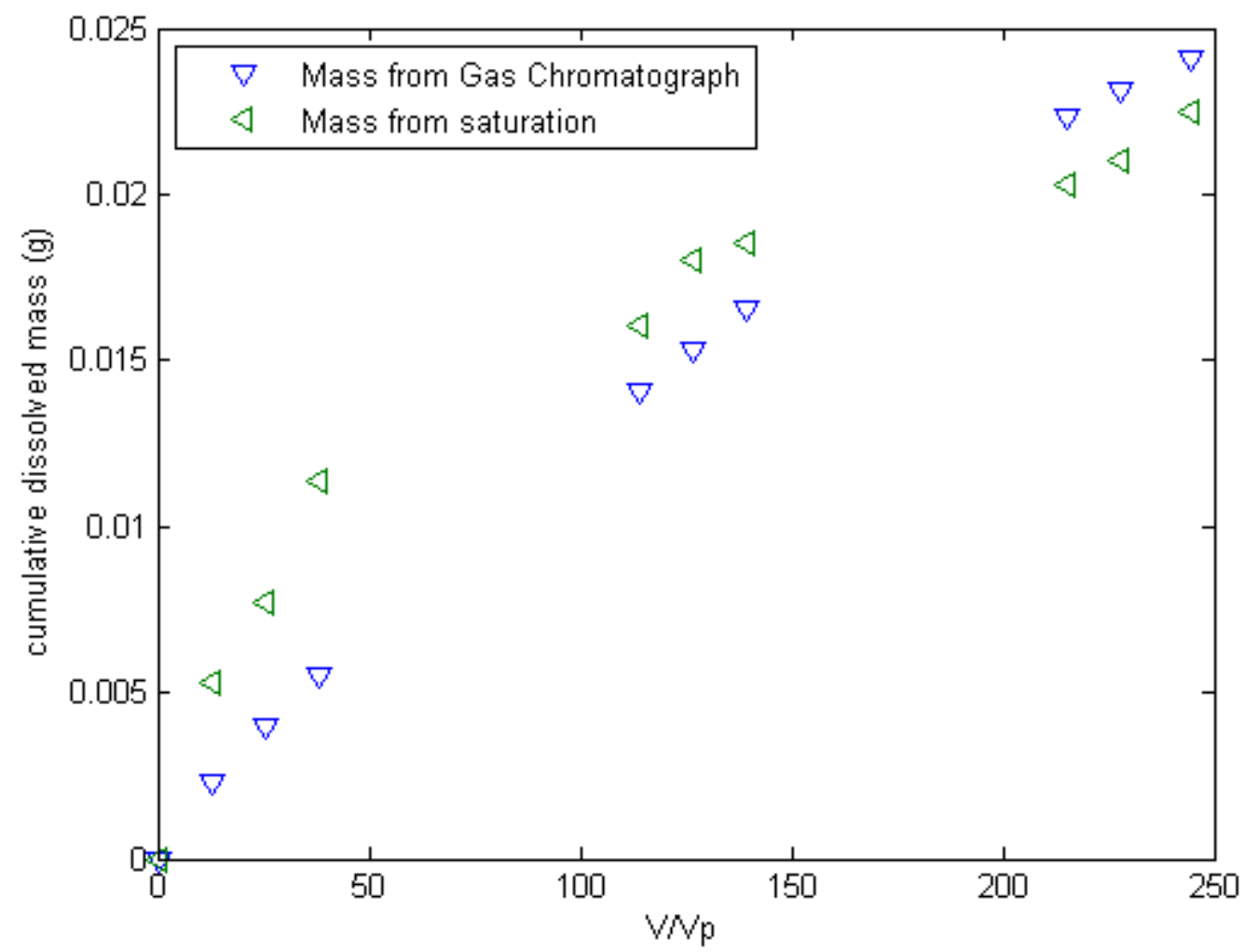

Figure 6: Mass balance of dissolved toluene as a function of the number of pore volumes injected $\left(V / V_{p}\right)$ for $P_{e}=22$

\subsubsection{Effect of Péclet number on the dissolution of toluene}

Dissolution-driven changes in saturation with time is shown in Fig. 7 for both experiments $\left(P_{e}=7\right.$ and $\left.P_{e}=22\right)$. To facilitate this comparison, the results are presented in terms of effective saturation. The analysis of this figure indicates a global decrease of oil saturation for the two experiments but with significant differences. First, a sharp increase of oil saturation is observed after 20 injected pore volumes for the low- Péclet experiment caused by the entrance of an oil blob from the inlet reservoir within the domain. In addition, for Experiment II (high Péclet number), the oil saturation decrease is faster and more significant (for $V / V_{p}<50$ ). These results show that the dissolution rate increases with increasing number of Péclet in accordance with the results of Corapcioglu and Baehr (1987), Miller et al. (1990), Miller and Poirier-Mcnell (1990) and Corapcioglu et al. (2009b). 




Figure 7: Changes in NAPL saturation as a function of the number of pore volumes injected $\left(P_{e}=7\right.$ and $\left.P_{e}=22\right)$

The elution curves of toluene concentration at the outlet of the flow cell are shown in Figs. 8 and 9, In both conditions, the curves exhibit three distinct dissolution regimes (Yra et al., 2006; Borden and Kao, 1992): an equilibrium regime (step 1), an exponential decay of the concentration (step 2) and an asymptotic regime (step 3).

- equilibrium regime: During this period, the toluene concentrations are constant and close to the equilibrium concentration. As expected, given the range of Péclet numbers of interest (convection-dominated flow), concentrations depart quickly from equilibrium, enhanced by the onset of preferential channels of dissolution. At low Péclet (Experiment I), with the exception of the second value, greater than 1 and which can be attributed to measurement error, the toluene concentration remains close to the equilibrium value for approximately $20 \mathrm{~V} / V_{p}$. At high Péclet, this step is difficult to delimitate. In any case, it is no longer than $10 \mathrm{~V} / \mathrm{V}_{p}$.

- exponential decay dissolution regime: This step is characterized by a quick decay of toluene concentration. The flux of dissolved mass from the NAPL droplets is no longer sufficient to maintain a toluene concentration close to equilibrium within the 
aqueous phase. Since the flow cell is continuously flushed with water, the averaged toluene concentration in the fluid bulk drops quickly.

- asymptotic regime: At this point, mass exchange flux with the NAPL phase has significantly reduced since the isolated blobs of oil present within the preferential flow channels have already been dissolved. This asymptotic regime is marked by a tailing effect on the toluene dissolution curve and this step is particularly visible for the experiment carried out at low Péclet (Experiment I). The toluene concentration values are significantly lower than the equilibrium concentration and the content of toluene extracted from the medium is very low compared to the previous step.

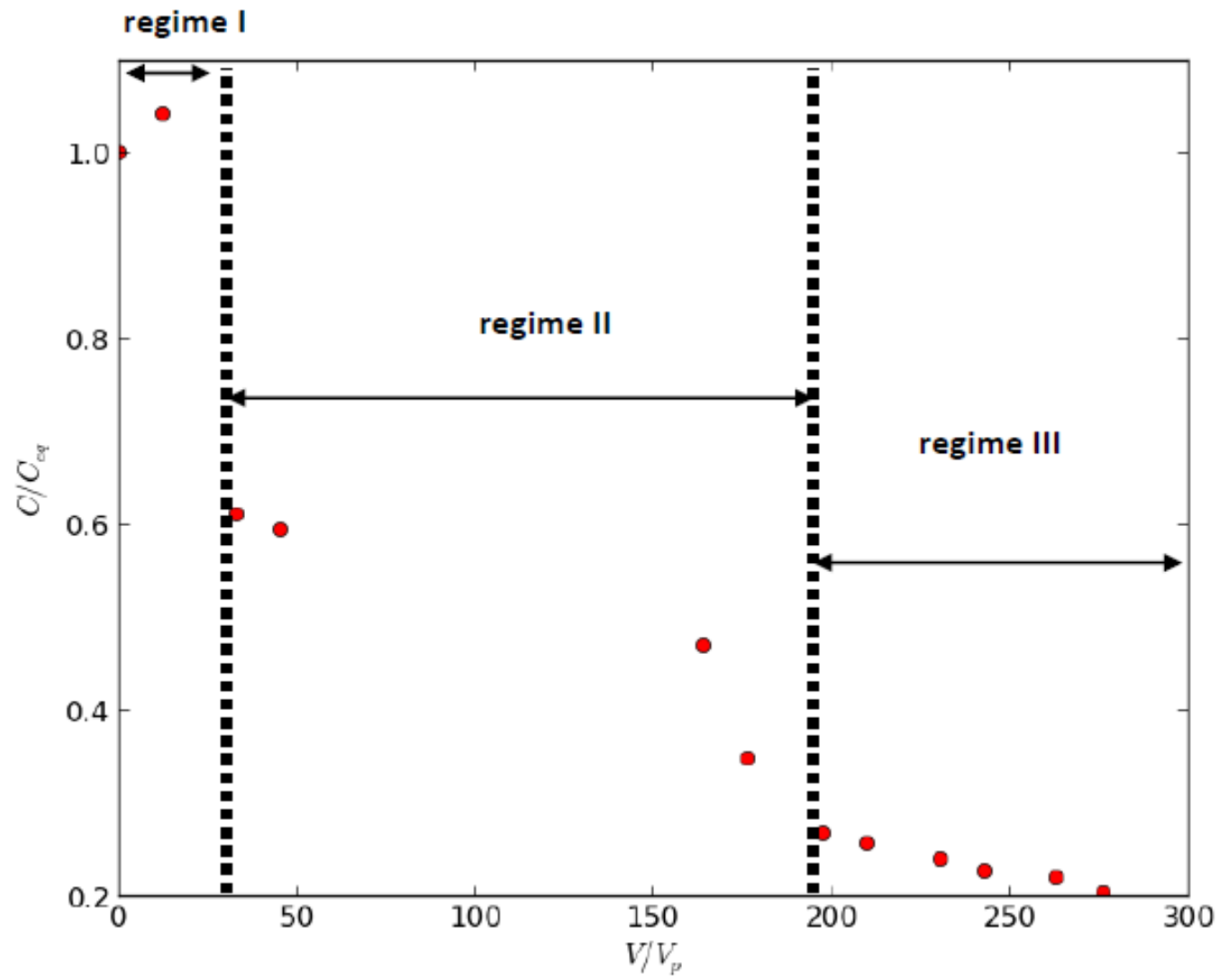

Figure 8: Normalized effluent concentration $\left(C / C_{e q}\right)$ versus pore volumes of water injected $\left(V / V_{p}\right)$ for $P_{e}=7$ 




Figure 9: Normalized effluent concentration $\left(C / C_{e q}\right)$ versus pore volumes of water injected $\left(V / V_{p}\right)$ for $P_{e}=22$

\section{Theoretical approach}

\subsection{Pore scale problem}

The pore scale problem (Fig. 10) under consideration corresponds to the mass balance of a component $A$ within a two-phase system, respectively the fluid $(\beta)$ and the NAPL $(\gamma)$ phases. The $\gamma$ - phase is assumed trapped residually into the porous matrix and it is immobile (e.g., Quintard and Whitaker, 1994, 1999). In the $\beta$-phase, solute transport is governed by advection and diffusion. We restrict our analysis to the simplest possible process in which the $\gamma$-phase consists of a single component (toluene denoted by species $A$ ) that is slightly soluble in the $\beta$-phase so that it can be considered as a tracer dissolved in the aqueous phase (Quintard and Whitaker, 1999). We do not consider here the problem of flow and velocity field is assumed to be known.

The pore-scale boundary value problem under consideration is described by the following microscopic equations: 


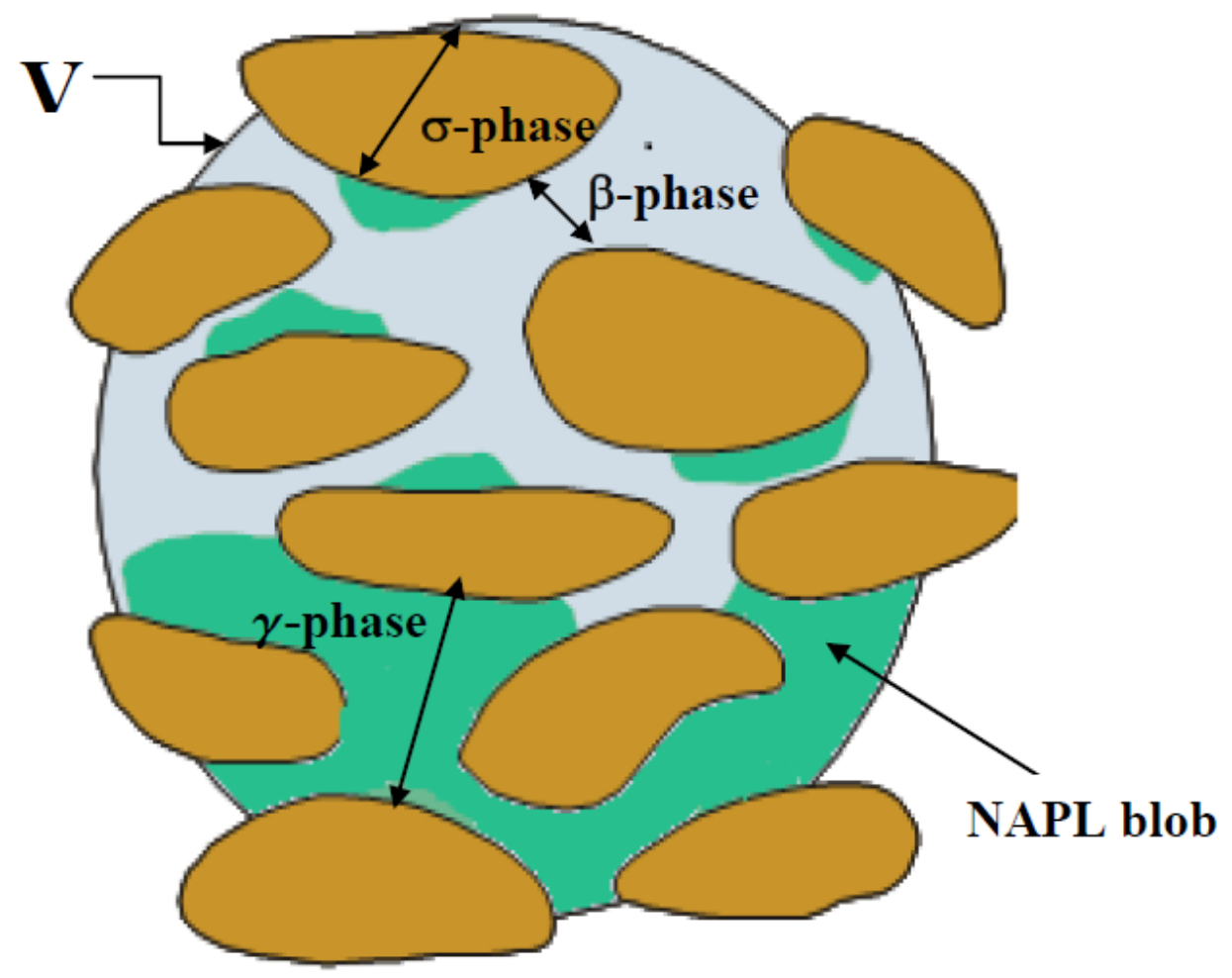

Figure 10: Representative elementary volume and associated phases

\section{Momentum equation}

$$
\nabla p_{\beta}=\mu_{\beta} \nabla^{2} \mathbf{v}_{\beta} \quad \text { in the } \beta \text {-phase }
$$

\section{Mass balance equations}

$$
\begin{aligned}
& \qquad \frac{\partial\left(c_{A \beta}\right)}{\partial t}+\nabla \cdot\left(\mathbf{v}_{A \beta} c_{A \beta}\right)=0 \quad \text { in the } \beta \text {-phase } \\
& \qquad \frac{\partial \rho_{\gamma}}{\partial t}=0 \quad \text { in the } \gamma \text {-phase } \\
& \text { B.C. } 1 \quad c_{A \beta}\left(\mathbf{v}_{A \beta}-\mathbf{w}_{\beta \sigma}\right) \cdot \mathbf{n}_{\beta \sigma}=0 \quad \text { at } A_{\beta \sigma} \\
& \text { B.C. } 2 c_{A \beta}=K_{\beta \gamma} \rho_{\gamma}=c_{A \beta}^{e q} \quad \text { at } A_{\beta \gamma} \\
& \text { B.C. } 3 c_{A \beta}\left(\mathbf{v}_{A \beta}-\mathbf{w}_{\beta \gamma}\right) \cdot \mathbf{n}_{\beta \gamma}=-\rho_{\gamma} \mathbf{w}_{\beta \gamma} \cdot \mathbf{n}_{\beta \gamma} \quad \text { at } A_{\beta \gamma} \\
& \text { B.C. } 4 \quad \mathbf{v}_{\beta}=0 \quad \text { at } A_{\beta \sigma} \\
& \text { B.C.5 } \quad \mathbf{v}_{\beta}=0 \quad \text { at } A_{\beta \gamma}
\end{aligned}
$$

Here, $c_{A \beta}$ represent the mass concentration of component $\mathrm{A}$ in the fluid phase; $\rho_{\gamma}$ is the mass concentration of pure species $\mathrm{A}$ in the $\gamma$ phase; $\mathbf{v}_{A \beta}$ represent the velocity of component $\mathrm{A}$ 
in the $\beta$ phase and $\mathbf{v}_{\beta}$ the mass average fluid velocity; $c_{A \beta}^{e q}$ is the concentration of species $A$ in the $\beta$ - phase in equilibrium with $\gamma$;

In addition, we determine the volumetric rate of dissolution of the NAPL phase by arranging the form of Eq.(7) as:

$$
\mathbf{w}_{\beta \gamma} \cdot \mathbf{n}_{\beta \gamma}=-\frac{1}{\rho_{\gamma}} c_{A \beta}\left(\mathbf{v}_{A \beta}-\mathbf{w}_{\beta \gamma}\right) \cdot \mathbf{n}_{\beta \gamma} \quad \text { at } A_{\beta \gamma}
$$

where $\mathbf{w}_{\beta \gamma}$ is the velocity of the fluid-NAPL interface $A_{\beta \gamma}$. Under these assumptions, the pore-scale problem is similar to the one treated by Quintard and Whitaker (1994).

\subsection{Volume averaging}

As mentioned in the introduction, our upscaling approach is based on the volume averaging method. The goal of this multiscale analysis is to compare these results with laboratory experiments; upscaling is necessary here to connect the micro-scale information with the behavior at the macroscale. In this present study, we recall the main definitions that have allowed the development of the macro-scale model. Referring to the method of volume averaging Whitaker (1999), we define the superficial average concentration of species $A$ in the $\beta$-phase as:

$$
\left\langle c_{A \beta}\right\rangle=\frac{1}{V} \int_{\vartheta_{\beta}(x, t)} c_{A \beta} d V
$$

with $V$, describing the averaging volume (the averaging domain is a geometric entity of macroscopic field $\left.\mathbb{V}_{\mathbb{M}}\right)$ and $\vartheta_{\beta}(x, t)$ is the Euclidean space representing the $\beta$-phase contained in the volume $V$. The intrinsic average concentration for the $\beta$-phase is given by:

$$
\left\langle c_{A \beta}\right\rangle^{\beta}=\frac{1}{V_{\beta}(x, t)} \int_{\vartheta_{\beta}(x, t)} c_{A \beta} d V
$$

with $V_{\beta}(x, t)$ is the Lebesgue measure of $\vartheta_{\beta}(x, t)$, that is, the volume of the $\beta$ phase. Superficial and intrinsic averages given by Eqs.(11) and (12) are related by:

$$
\left\langle c_{A \beta}\right\rangle=\epsilon_{\beta}(x, t)\left\langle c_{A \beta}\right\rangle^{\beta}
$$

where $\epsilon_{\beta}(x, t)$ represents the volume fraction of the $\beta$-phase defined as:

$$
\epsilon_{\beta}(x, t)=\frac{V_{\beta}(x, t)}{V}
$$

In the development of the model, the pore-scale concentration $c_{A \beta}$ can be expressed following Gray's decomposition (Gray et al., 1993):

$$
c_{A \beta}=\left\langle c_{A \beta}\right\rangle^{\beta}+\tilde{c}_{A \beta}
$$

with $\tilde{c}_{A \beta}$ the spatial deviation concentration in $\beta$ phase. 
The main steps of the derivation of the macroscopic balance equations are reminded in AppendixA and we refer the reader to Quintard and Whitaker (1994) for more details (see also Bahar et al. (2016) for an extension of this problem to bio-enhanced NAPL dissolution). This upscaling leads to the following non-equilibrium model of NAPL dissolution:

$$
\begin{gathered}
\frac{\partial \epsilon_{\beta}\left\langle c_{A \beta}\right\rangle^{\beta}}{\partial t}+\epsilon_{\beta}\left\langle\mathbf{v}_{\beta}\right\rangle^{\beta} \cdot \nabla\left\langle c_{A \beta}\right\rangle^{\beta}-\mathbf{d}_{\beta \omega} \cdot \nabla\left\langle c_{A \beta}\right\rangle^{\beta}-\mathbf{U}_{\beta \omega} \cdot \nabla\left\langle c_{A \beta}\right\rangle^{\beta}= \\
\nabla \cdot\left(\mathbf{D}_{\beta \omega}^{*} \cdot \nabla\left\langle c_{A \beta}\right\rangle^{\beta}\right)+\alpha_{A}^{*}\left(c_{A \beta}^{e q}-\left\langle c_{A \beta}\right\rangle^{\beta}\right) \\
\rho_{\gamma} \frac{\partial \epsilon_{\gamma}}{\partial t}=-\alpha_{A}^{*}\left(c_{A \beta}^{e q}-\left\langle c_{A \beta}\right\rangle^{\beta}\right)
\end{gathered}
$$

where $\mathbf{D}_{\beta \omega}^{*}$ (Darcy scale effective diffusion tensor for the species $A$ ), $\alpha_{A}^{*}$ (mass-transfer coefficient for the species $A$ ), $\mathbf{d}_{\beta \omega}$ and $\mathbf{U}_{\beta \omega}$ (convective-like effective transport coefficients) are the effective transport coefficients. Note that the mass transfer exhibited here is a lumped mass transfer coefficient that incorporates the surface area per unit porous medium volume, as classically defined in the Gilland-Sherwood correlations. Effective coefficients which appear in these equations are given explicitly by a set of two closure problems which have been introduced by Quintard and Whitaker (1994) and are presented in AppendixA. The resulting macroscopic model describes NAPL dissolution and transport of the dissolved contaminant at the Darcy scale. Finally, the macroscopic equations can be rewritten in a most convenient form (1D dimensionless equations) that we will use later (where $S h^{\prime}$ is the modified Sherwood number):

\section{Fluid}

$$
\frac{\partial \epsilon_{\beta}\left\langle c_{A \beta}\right\rangle^{\prime}}{\partial t}+\left(\epsilon_{\beta} P_{e}-\mathbf{d}_{\beta \omega}^{\prime}-\mathbf{U}_{\beta \omega}^{\prime}\right) \frac{\partial\left\langle c_{A \beta}\right\rangle^{\prime}}{\partial x}=\mathbf{D}_{\beta \omega}^{*^{\prime}} \frac{\partial^{2}\left\langle c_{A \beta}\right\rangle^{\prime}}{\partial x^{2}}+S h^{\prime}\left(1-\left\langle c_{A \beta}\right\rangle^{\prime}\right)
$$

NAPL

$$
\rho_{\gamma}^{\prime} \frac{\partial \epsilon_{\gamma}}{\partial t^{\prime}}=-S h^{\prime}\left(1-\left\langle c_{A \beta}\right\rangle^{\prime}\right)
$$

where

$$
\begin{array}{r}
\left\langle c_{A \beta}\right\rangle^{\prime}=\frac{\left\langle c_{A \beta}\right\rangle^{\beta}}{c_{A \beta}^{e q}} \quad \mathbf{D}_{\beta \omega}^{*^{\prime}}=\frac{\mathbf{D}_{\beta \omega}^{*}}{D_{A \beta}} \quad \mathbf{d}_{\beta \omega}^{\prime}=\frac{\mathbf{d}_{\beta \omega} l_{\beta}}{D_{A \beta}} \quad \mathbf{U}_{\beta \omega}^{\prime}=\frac{\mathbf{U}_{\beta \omega} l_{\beta}}{D_{A \beta}} \\
S h^{\prime}=\frac{\alpha_{A}^{*} l_{\beta}^{2}}{D_{A \beta}} \quad t^{\prime}=\frac{t D_{A \beta}}{l_{\beta}^{2}} \quad \rho_{\gamma}^{\prime}=\frac{\rho_{\gamma}}{c_{A \beta}^{e q}}
\end{array}
$$

In this work, we use COMSOL Multiphysics Software ${ }^{\circledR}$ to numerically solve the equations presented above. The computer code is based on the finite element method for solving various physical problems defined by partial differential equations (PDE). The first 
step is to calculate the effective parameters from solving two closure problems associated with the macroscopic model on the representative unit cell of the porous medium. The resolution method of these closure problems is the same as detailed by Bahar et al. (2016). The second step is solving the macroscopic equation on the entire domain. The macroscopic problem is one-dimensional and transient. Ultimately, we obtain the changes in concentration versus time (breakthrough curve) that we compare to the experimental curve of residual toluene dissolution.

\subsection{Calculation of effective parameters and historicity effect}

The effective parameters of the macroscopic model are represented by the coefficients $\mathbf{D}_{\beta \omega}^{*}, \mathbf{d}_{\beta \omega}, \alpha_{A}^{*}$ and $\mathbf{U}_{\beta \omega}$ which are dependent only of the closure problems (Bahar et al., 2016). The most driving effective coefficients are the effective dispersion and mass transfer coefficient. The additional terms $\left(\mathbf{d}_{\beta \omega}\right.$ and $\left.\mathbf{U}_{\beta \omega}\right)$ represent the terms of non-traditional convection varying relatively low and can be usually neglected relative to the classical convection term $\left\langle\mathbf{v}_{\beta}\right\rangle$ in first approximation (Quintard and Whitaker, 1994).

The underlying difficulty here is that the solution of closure problems requires that the geometry of the fluid-solid interface is known. However, this interface may change in a complex manner during the dissolution process resulting in changes in the indicator phase and the same time in porosity. In other words, the effective properties can depend on the process of dissolution by path-dependency and evolve in a complex manner as a function of time. In this work, we adopt the traditional approach and we approximate the historical behavior of the system by considering a direct macroscopic relationship between macroscopic variables. It is often assumed, in the problems of dissolution for instance, for a given sample of rock, the existence of a direct correlation connecting permeability $(\mathrm{K})$ to porosity $(\epsilon)$, i.e., $K(\epsilon)$ instead of $K(t)$ and $\epsilon(t)$. The most notable example of such a relationship is the Kozeny-Carman equation. In the following, based on the observed relationships at Darcy scale and the form of the closure problems (Bahar et al., 2016), we will express the assumption that the mass transfer coefficient $\alpha_{A}^{*}$ and the longitudinal dispersion coefficient depend on both the pore Péclet number $P_{e}$ and the volume fraction of NAPL $\epsilon_{\gamma}$ (or the saturation $S_{\gamma}$ ). Due to the weak variation of the pore fluid velocity during the experiments, we will consider the dependency to Péclet number is negligible compared to the influence of $\epsilon_{\gamma}$. Note that this approach has been already used with success for acid dissolution in porous media (Golfier et al., 2002) and similar situations are observed in dendrite structures problems (Goyeau et al., 1999).

We determine the dimensionless effective parameters (dispersion coefficient and mass transfer coefficient) of our macroscopic equation by the following relationships

$$
\begin{aligned}
\frac{\mathbf{D}_{\beta \omega}^{*}}{D_{A \beta}}=\epsilon_{\beta} \mathbf{I}-P_{e A}\left\langle\tilde{\mathbf{v}}_{\beta}^{\prime} \mathbf{b}_{\beta}^{\prime}\right\rangle+\left(\frac{1}{V} \int_{A_{\beta \gamma}} n_{\beta \gamma} \mathbf{b}_{\beta}^{\prime} d A+\frac{1}{V} \int_{A_{\beta \sigma}} n_{\beta \sigma} \mathbf{b}_{\beta}^{\prime} d A\right) \\
\frac{\alpha_{A}^{*} L^{2}}{D_{A \beta}}=\frac{1}{V} \int_{A_{\beta \gamma}} n_{\beta \gamma} \nabla \mathbf{s}_{\beta}^{\prime} d A
\end{aligned}
$$


where $\mathbf{b}_{\beta}^{\prime}$ and $\mathbf{s}_{\beta}^{\prime}$ are the closure variables, solutions of the closure problems $I$ and $I I$ (Eqs. A.18 A.23 and Eqs. A.24 A.29 in AppendixA.

\section{Comparisons and discussions}

Firstly, we present the results of calculation of effective parameters based on the unit cells reconstructed from the images of the experimental flow cell (micromodel). These realistic geometries are expected to capture most of the microscopic geometrical features of the porous medium. The dissolution of toluene with time induced spatio-temporal changes of these properties (e.g., toluene saturation, volume fractions of the phases) and a correct prediction requires considering the experiments and all of their variability. Our experimental set-up offers us the benefit of having images of the process of dissolution versus time which subsequently will be processed for obtaining realistic configurations. In a second step, the macroscopic equations will be solved on the entire domain (1D domain).

\subsection{Selection and construction of unit cells}

The unit cells constructed from the images of the experimental micromodel are shown in Fig. 11. The unit cell (A) shows the initial state of micromodel (image of the residual saturation in toluene at $t=0$ hour), the unit cell (B) was imaged after two hundred hours of dissolution (residual saturation at $t=200$ hours) and finally the unit cell (C) illustrates the final state of the experiment after three hundred sixty-seven hours of dissolution (residual saturation at $t=367$ hours). These three representative images are chosen to approximate the historical behaviour of the dissolution process due to the variation over time of the geometry and by the same of the effective parameters. The main physical parameters associated with the different unit cells are summarized in Table 2. Morphological analysis conducted on these geometries (see Table 2) give interesting insights on the changes in pore-scale features captured by these unit cells. Feret diameter (also known as maximum caliper) measures the longest distance between any two pixels along the NAPL boundary and circularity is a compactness measure of the shape of NAPL phase (a value of 1.0 indicates a perfect circle). Lacunarity is measured from digital images using the box counting method and indicates the coefficient of variation (CV) for the number of NAPL pixels per box. Wwe refer the reader to the ImageJ documentation (Rasband, 1997-2016) for more details about their calculation. The decrease of maximal Feret diameter for the non-wetting phase is consistent with the progressive dissolution of NAPL ganglia with time. The similar trend for lacunarity that indicates a decrease of heterogeneity support this finding and emphasizes a preferential dissolution of the smallest isolated NAPL blobs. This preferential dissolution mechanism is consistent with the increase of compacity of the oil phase as indicated by the circularity value for unit cell (B). Note that these unit cells are extracted from experiments at low Péclet number. For the calculation at high Péclet, we keep the same unit cells, considering that the dissolution patterns observed have a similar behavior.

The construction of these unit cells is based on a procedure combining several image processing and analysis tools. The first step consists in extracting the active part of the 
flow cell by eliminating the upstream and downstream reservoirs via the ImageJ tool (Figure 12. . Next, the Adobe tool is used to binarize and filter the geometric elements of the image in order to recover the maximum of the edges and boundaries representing the phases. The final step in the process consists of reconstructing the cells and the phase connectivity from the previous images under Comsol Multiphysics. It is important to note that during the construction of cells, information losses (underestimation of the residual saturation for example) occured during image processing (due to thresholding and reconstruction of geometry in Comsol Multiphysics). We have also kept as much as possible the porosity of the cell around $50 \%$ but in spite of our efforts, a part of the smallest grains, especially in the highly contamined areas, were lost and the resulting porosity for the unit cells is a little bit higher than the experimental measurement, i.e. 0.58 against $0.5 \pm 0.02$. To illustrate this loss of fidelity, we represent in Figure 12 the main steps of construction of the unit cell (A) and the protocol is identical for the other two cells (unit cell (B) and (C)). We can clearly see the geometrical features lost during the various steps of protocol. We have quantified the saturation variations between the total initial image, the extracted image and the final cell constructed for all the unit cells. Results are summarized in Table 2. However, the error induced by image processing has not a significant effect on the calculation of the averaged properties. When we conduct some form of upscaling, indeed, we seek to only maintain the important features of the physical and chemical processes (pore size, various heterogeneities, distribution of volume fractions, phase connectivity, ...) expressed at the smallest scale of the problem. Globally the unit cells obtained meet these criteria despite some underestimation. 



Unit cell(B)

Figure 11: Unit cells constructed from the images of the experimental cell 




Figure 12: Representation side by side of the initial image used (image of residual saturation at $t=0$ ), the image extracted and the unit cell built from this image.

\subsection{Calculation of the effective properties}

The calculation of effective properties is based on unit cells shown in Figure 11. Closure problems 1 and 2 are solved for each unit cell at high and low Péclet number and results for the two effective coefficients are reported as a function of the NAPL volume fraction. It should be emphasized that the values of effective coefficients obtained can be significantly different from those that could be measured experimentally (e.g., back-calculated values using image analysis). The solution given by the closure problem corresponds indeed to the asymptotic conditions. The choice of the unit cell is for this reason quite significant: if the choice of periodic conditions is perfectly appropriate far from boundary conditions, it becomes debatable when getting close macroscopic limits of the domain (cf. the paper of Cushman and Moroni (2001) in particular on the influence of non-local effects on the dispersion tensor). On the other hand, the dispersion tensor and the mass transfer coefficient are parameters inherently dependent of the scale of observation and their experimental 
Table 2: $\quad$ Physical parameters associated with the different unit cells

\begin{tabular}{lccc}
\hline \multirow{2}{*}{ Parameter } & \multicolumn{3}{c}{ Unit cells } \\
\cline { 2 - 4 } & $(\mathrm{A})$ & $(\mathrm{B})$ & $(\mathrm{C})$ \\
\hline Fluid volume fraction $\epsilon_{\beta}$ & 0.42 & 0.48 & 0.57 \\
\hline NAPL volume fraction $\epsilon_{\gamma}$ & 0.16 & 0.1 & 0.025 \\
\hline Characteristic length $L(\mathrm{~cm})$ & 3.7 & 3.7 & 3.7 \\
\hline $\begin{array}{l}\text { Relative difference in NAPL saturation between } \\
\text { the initial and extracted images (in } \%)\end{array}$ & 19.4 & 3.4 & 1.3 \\
\hline $\begin{array}{l}\text { Relative difference in NAPL saturation between } \\
\text { the extracted image and the unit cell }(\mathrm{A})(\text { in } \%)\end{array}$ & 5.7 & 22.5 & 54 \\
\hline Feret diameter $D_{f}(\gamma$ - phase) $(\mathrm{cm})$ & 1.17 & 1 & 0.86 \\
\hline Circularity $C_{f}(\gamma$ - phase $)$ & 0.28 & 0.36 & 0.21 \\
\hline Lacunarity $\lambda_{f}(\gamma$ - phase) & 0.16 & 0.18 & 0.1 \\
\hline
\end{tabular}

measures can be significantly affected by space (boundary effect, heterogeneity) and time (transient behavior) disturbances. For instance, a column-scale mass transfer coefficient would be significantly different from the Darcy-scale mass transfer coefficient predicted here. In other words, the values calculated for these parameters on the domain (via the choice of unit cells) can be different from those measured locally (particularly in view of the strong spatial variability of the initial toluene saturation) but give the best fit of effective properties required for an averaged description.

Further concerns may be raised, however, about the applicability of the volume averaging method (and more generally of any upscaling method) to this experiment and the definition of the Representative Elementary Volume (REV). The constraint of separation of scales needs, indeed, that the REV size is sufficiently large relative to the pore-scale heterogeneities but if such an REV exists, nothing forbids from using a larger unit cell than required. From a correlation length analysis, we have found that the pore characteristic length of the reconstructed porous medium - in the absence of NAPL - is about $0.08 \mathrm{~L}$, which can be used as a rough estimate for the minimum REV length. Assuming a length ratio of ten between the REV size and the pore-scale characteristic length is enough in general to satisfy the separation of scale condition for such a comparison between direct numerical and macro-scale simulation which means that the full domain can be used as REV. Using the complete domain as REV can seem useless since it is usually preferable to search for an unit cell that tends to minimize the amount of complexity needed to recover the essential physics of the process of interest. But the only objective of the present paper is to investigate the capability of an upscaled model to describe NAPL dissolution with accuracy. In other words, our only focus is to assess the theory. However, for high NAPL saturations, the 
pore-scale characteristic length can be larger than $0.08 \mathrm{~L}$ and the size of the domain might be not sufficient to ensure the separation of scales. It means that the asymptotic values of the effective coefficients (longitudinal dispersion, mass transfer coefficient) as predicted by the closure problem could underestimate the averaged behavior of the experimental domain at very short times. Upscaling theories requires also that spatial localization constraints are imposed upon averaged fields. Roughly speaking, it means in our case that the averaged spatial concentration gradients are small enough at the REV scale. Occurence of a very sharp macroscopic reaction front that could break down the validity of our upscaled theory is driven by conditions at both local and global scales but it usually requires that the macroscopic Damköhler number (calculated from the mass transfer coefficient value) is very large compared to the macroscopic Péclet number and it does not correspond to the current experimental conditions. In any case, no assumption is needed on the microscale concentration gradients. We do not use in the present study, indeed, an local mass equilibrium assumption that implies that microscale gradients are relatively small, so that both phases can be treated in exactly the same way. On the contrary, a local non-equilibrium model is considered so that strong concentration gradients may exist at the NAPL/fluid interface

\subsubsection{Variation of dispersion coefficient}

We show in Figure 13 the changes in the dimensionless longitudinal dispersion coefficient depending on the volume fraction of toluene for the two experiments of dissolution $\left(P_{e}=7\right.$ et $P_{e}=22$ ). These results indicate that the impact of the geometry remains relatively low in this case. Consequently, for the one-dimensional numerical model, we will use a constant dispersion coefficient value given its relatively small change with time (at least in the range of oil saturation investigated). We will keep arbitrarily in our simulations at constant Péclet number the maximum value of the longitudinal dispersion encountered for the three saturation values. 


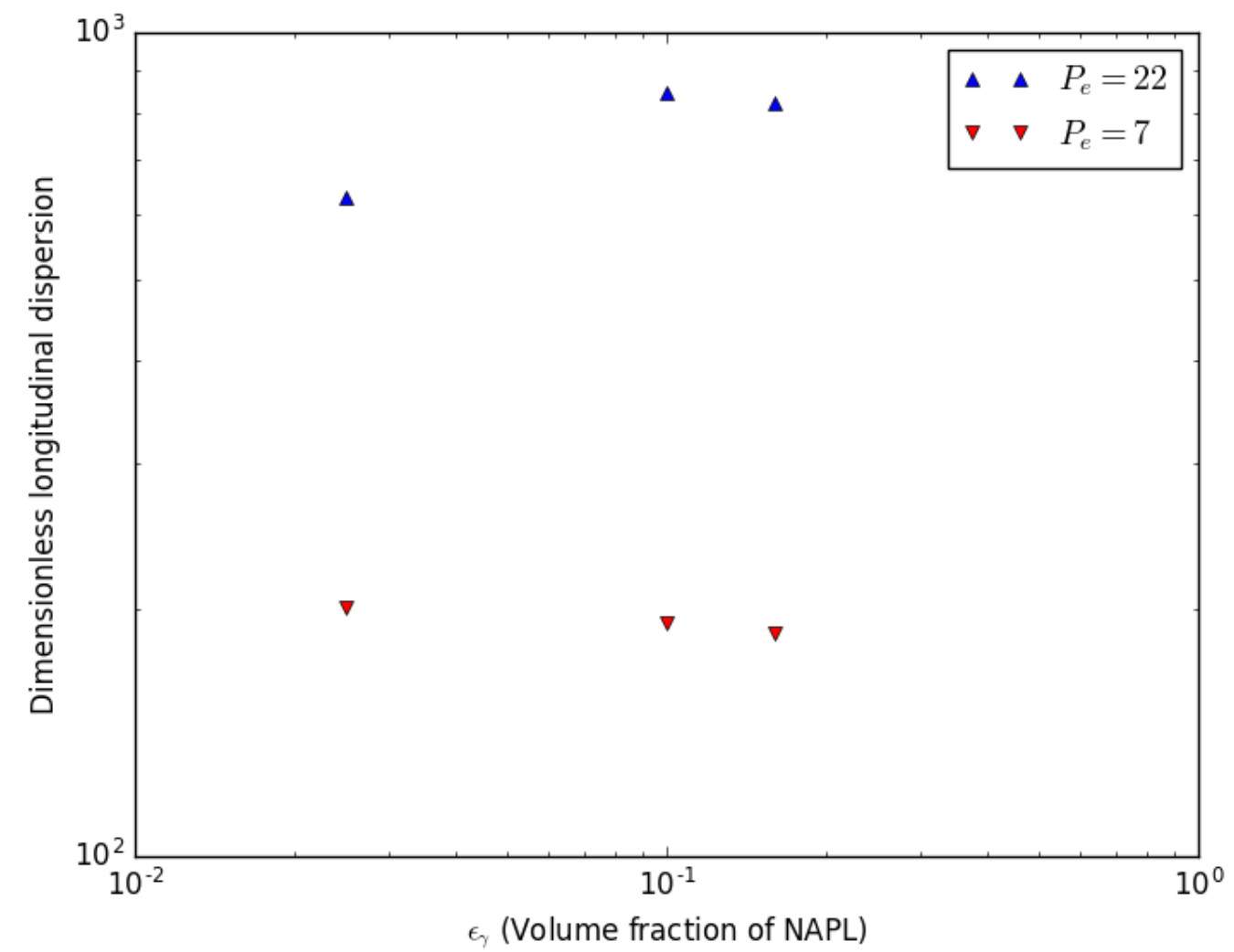

Figure 13: Evolution of dimensionless dispersion coefficient versus volume fraction of toluene for $P_{e}=7$ and $P_{e}=22$

\subsubsection{Variation of mass exchange coefficient}

The influence of the Péclet number and the toluene saturation on the mass exchange coefficient is presented in Fig. 14. Numerical calculations are conducted for three values of NAPL volume fraction $(0.16,0.1,0.025)$ and for Péclet numbers in the range of 0.001 to 1000. Results show overall an increase of mass transfer with toluene saturation and Péclet number. At low Péclet, the transfer of mass is mainly driven by the area of exchange between the pollutant and the aqueous phase. The area of exchange decreases during dissolution causing a reduction in the mass exchange coefficient (similar result has been obtained by Ahmadi et al. (2001) and Bahar et al. (2016) but for simplest units cells). The flow velocity in the vicinity of the fluid-NAPL interface decreases in parallel of the dissolution (due to decreasing oil saturation), which accentuates this difference between the initial (unit cell (A))and final geometry (unit cell $(\mathrm{C})$ ) in terms of mass transfer. Note that a similar trend has been observed by Miller et al. (1990). Our results confirm that the dissolution-induced changes on geometry may explain limitations on mass transfer. 


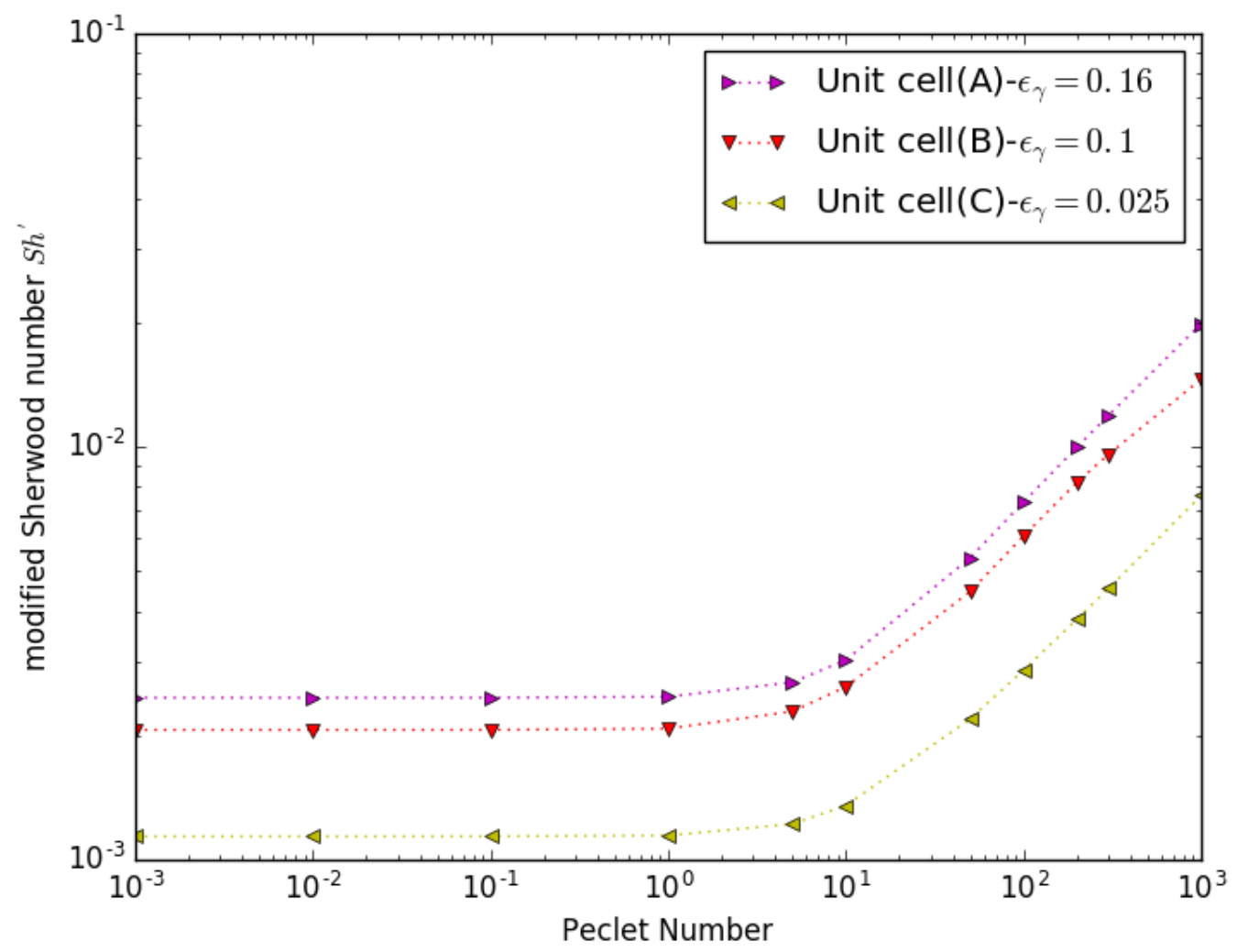

Figure 14: Dimensionless mass exchange coefficient: influence of Péclet number and toluene saturation

In a second step, we extract from these calculations the mass transfer coefficients values at different NAPL saturations for low- and high-Péclet experiments. For prediction of the experimental breakthrough curve, indeed, the numerical model requires knowledge of mass transfer coefficient. By keeping a constant value in the calculations, it is highly probable that the dissolution curve will not be correctly predicted. For that reason, we opted for a dependency of mass transfer coefficient (or modified Sherwood number) on the NAPL content, based on the numerical results obtained Fig. 14. The main advantage of this approach resides in the fact that the mass exchange coefficient is now correlated with the dynamics of the physical problem. Contrary to the empirical correlations valid for a specific range of hydrodynamic conditions, our upscaled mass transfer is expected to follow a physical behavior, i.e., the molecular diffusion prevails for low values of $\mathrm{Pe}(P e<1)$ and maintains a non-zero mass flux between the NAPL phase and the bulk fluid. As mentioned in the introduction, indeed, a large number of relationships expressing the modified Sherwood number in terms of Reynolds number, Schmidt number, Péclet number and the oil volume fraction are available in the literature. We list in Table 3 some of the most significant correlations (Miller et al., 1990; Nambi and Powers, 2003; Saba and Illangasekare, 2000) used for predicting the lumped mass transfer coefficient and their range of validity. 
Table 3: Dimensionless correlations for the modified Sherwood numbers (modified from Saba and Illangasekare (2000))

\begin{tabular}{ccc}
\hline Reference & Correlation & Range of validity \\
\hline Miller et al. (1990) & $12 \cdot S c^{0.5} \cdot R_{e}^{0.75} \cdot \epsilon_{\gamma}^{0.6}$ & $1 \mathrm{D}, R_{e}=0.05-0.1, S_{\gamma}=0 .-0.21$ \\
Saba and Illangasekare $(2000)$ & $11.34 \cdot S c^{0.33} \cdot R_{e}^{0.2767} \cdot\left(\frac{l_{\beta} \epsilon_{\gamma}}{\tau L}\right)^{1.037}$ & $2 \mathrm{D}, R_{e}=0.0015-0.01, S_{\gamma} \approx 0.22$ \\
Nambi and Powers $(2003)$ & $37.15 \cdot R_{e}^{0.75} \cdot S_{\gamma}^{0.24}$ & $2 \mathrm{D}, R_{e}=0.018-0.134, S_{\gamma}=0.01-0.35$ \\
\hline
\end{tabular}

Here, $S_{\gamma}$ and $\epsilon_{\gamma}$ represent respectively the saturation and fluid volume fraction of the NAPL-phase, $l_{\beta}$ the characteristic pore length, $L$ the characteristic length of the domain (i.e, the unit cell) and $\tau$ the tortuosity (fixed to 2). For comparison purposes, we confronted our theoretical predictions to these empirical correlations in Fig. 15 for $P_{e}=7$. Similar results hold for $P_{e}=22$. First, we note that the correlations issued from the literature vary over several orders of magnitude. This preliminary observation confirm the conclusions of several authors (e.g., Powers et al., 1992; Ahmadi et al., 2001) that such simple relationships are difficult to generalise to complex experimental conditions where NAPL-phase distribution and velocity may evolve in a very different way and a more accurate description of pore-scale features is mandatory to describe with accuracy the mass transfer processes. Second, we observe a very good agreement with the correlation of Saba and Illangasekare (2000) even if the gap seems more pronounced at low saturation values. This best correspondence compared with the other correlations - is not surprising since the range of validity of Saba and Illangasekare (2000) relationship is the closest of our experimental conditions (see Table 1) although the "2D medium" of Saba and Illangasekare (2000) refers to flow cells filled with beads and not micromodels. This agreement is also to be relativised since a difference of factor 2 to 3 remains with our calculations which is not negligible in terms of dissolved flux rates.

\subsection{Comparison between experimental results and numerical simulations}

To complete the calculations of effective coefficients, we still need to evaluate the nontraditional convective-like terms (i.e., correction terms for diffusive and convective fluxes). We followed the same procedure than previously for the two other macroscopic parameters. Results (not presented here) indicate a relatively small variation of these terms $\left(\mathbf{d}_{\beta \omega}\right.$ and $\mathbf{U}_{\beta \omega}$ ) as a function of the geometry tested (unit cells A, B and C) and in any cases, values remain weak in regard to the convective term. The boundary and initial conditions associated with the set of macroscopic governing equations, Eqs. 18 and 19, are detailed below and refer to experimental conditions. As mentioned in the experimental part, the micromodel is put at rest for twenty-four hours after the imbibition step. Taking into account the waiting period, we assume that at the initial time of the dissolution experiment, the aqueous phase put itself in equilibrium with the NAPL phase throughout the porous medium. Thus we have 


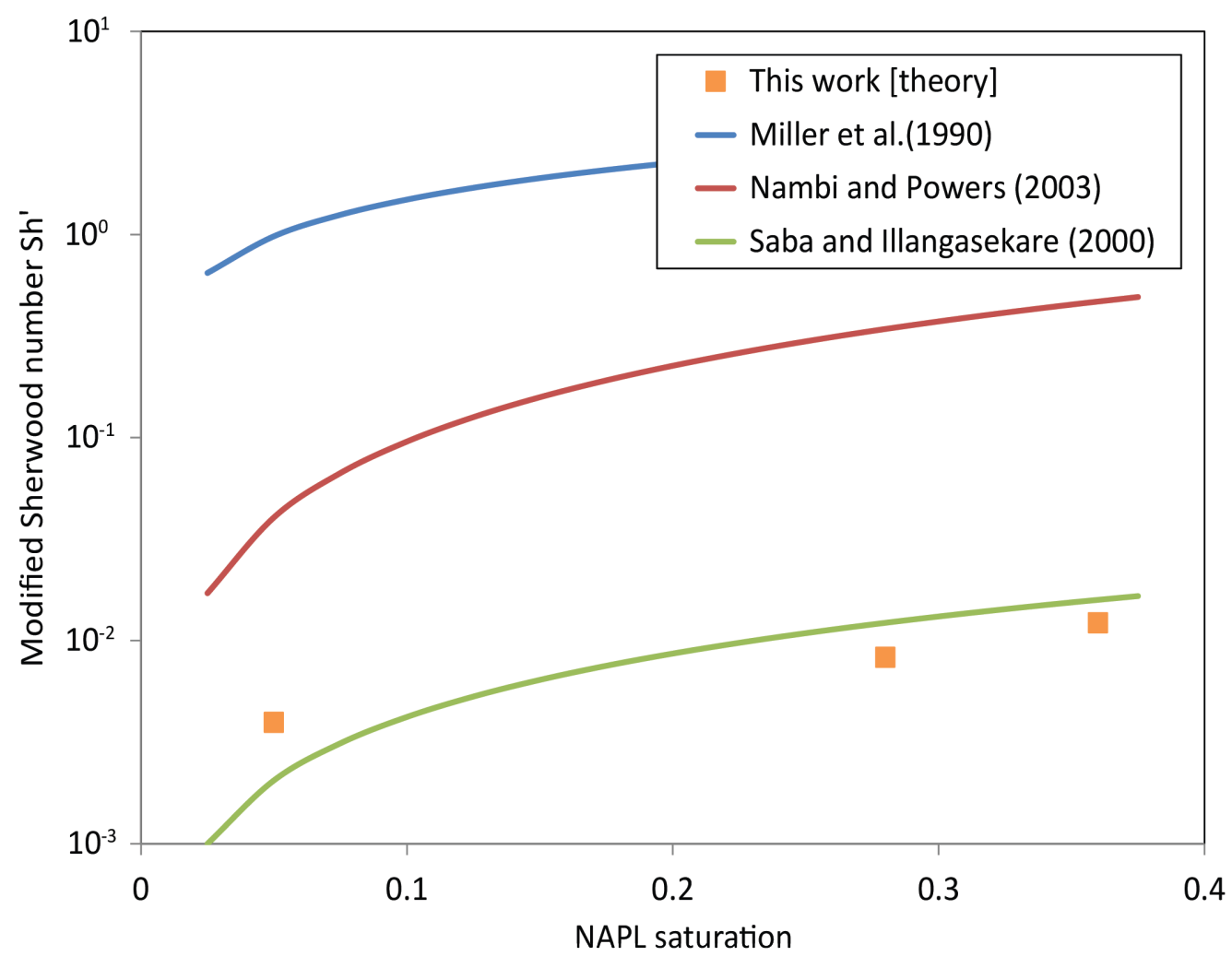

Figure 15: Variation of the modified Sherwood number versus the oil saturation for $P_{e}=7:$ comparison between theoretical and empirical correlations

at the inlet $(x=0): \quad\left\langle c_{A \beta}\right\rangle^{\prime}=0$,

$$
\begin{aligned}
\text { at the outlet }(x=L): & D_{\beta \omega}^{*} \frac{\partial\left\langle c_{A \beta}\right\rangle^{\prime}}{\partial x}=0, \\
\text { at } t=0: \quad & \left\langle c_{A \beta}\right\rangle^{\prime}=c_{A \beta}^{e q} \quad \text { and } \quad \epsilon_{\gamma}=\epsilon S_{\gamma r}
\end{aligned}
$$

where $S_{\gamma r}$ is the initial oil saturation and $\epsilon$ represents the porosity of porous medium. Finally, Darcy-scale simulations, given by Eqs. 18 and 19 , are conducted over a onedimensional equivalent domain of length equal to $L$. We would like to emphasize that the above-mentioned macro-scale model is intended to be used as a forward model. In other word, effective property values are directly extracted from solving closure problems and no parameter fitting was conducted to obtain a better agreement with the experimental data.

Figures 16 and 17 present the comparison between the experimental results and numerical simulations (for the two Péclet numbers). Examination of these figures shows that the one-dimensional model (at $P_{e}=7$ and $P_{e}=22$ ) describe satisfactorily the breakthrough curves observed experimentally. We recover quasi-immediately a non-equilibrium behavior in agreement with the experimental results (Figures 7 and 8 ) followed by a slow decrease of 
toluene recovery. If the long-term behavior is correctly predicted, we note yet, that the twostep dissolution mechanism identified in section 2.2 , is not captured by the model. This is particularly obvious at low-Péclet where the dissolution regime of exponential decay is more marked. We assume that this discrepancy should be attributed to the phenomenon of NAPL remobilisation which is not taken into account in the model. Experimental artefacts such as the presence of dead zones in the outlet reservoir could also affect our GC measurements.

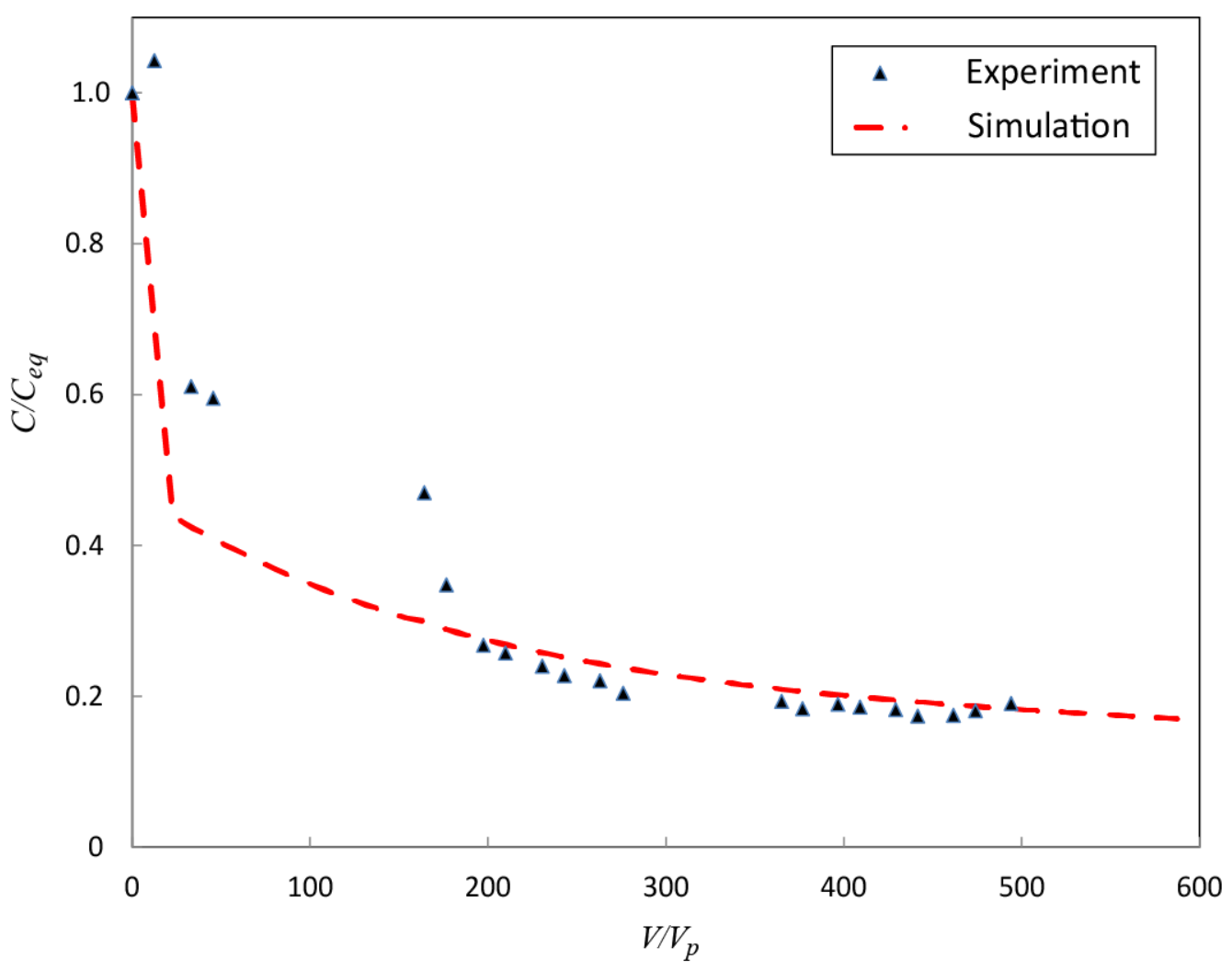

Figure 16: Normalized effluent concentration $\left(C / C_{e q}\right)$ versus pore volumes of water injected $\left(V / V_{p}\right)$ for $P_{e}=7$ (comparison between experimental results and numerical simulations) 


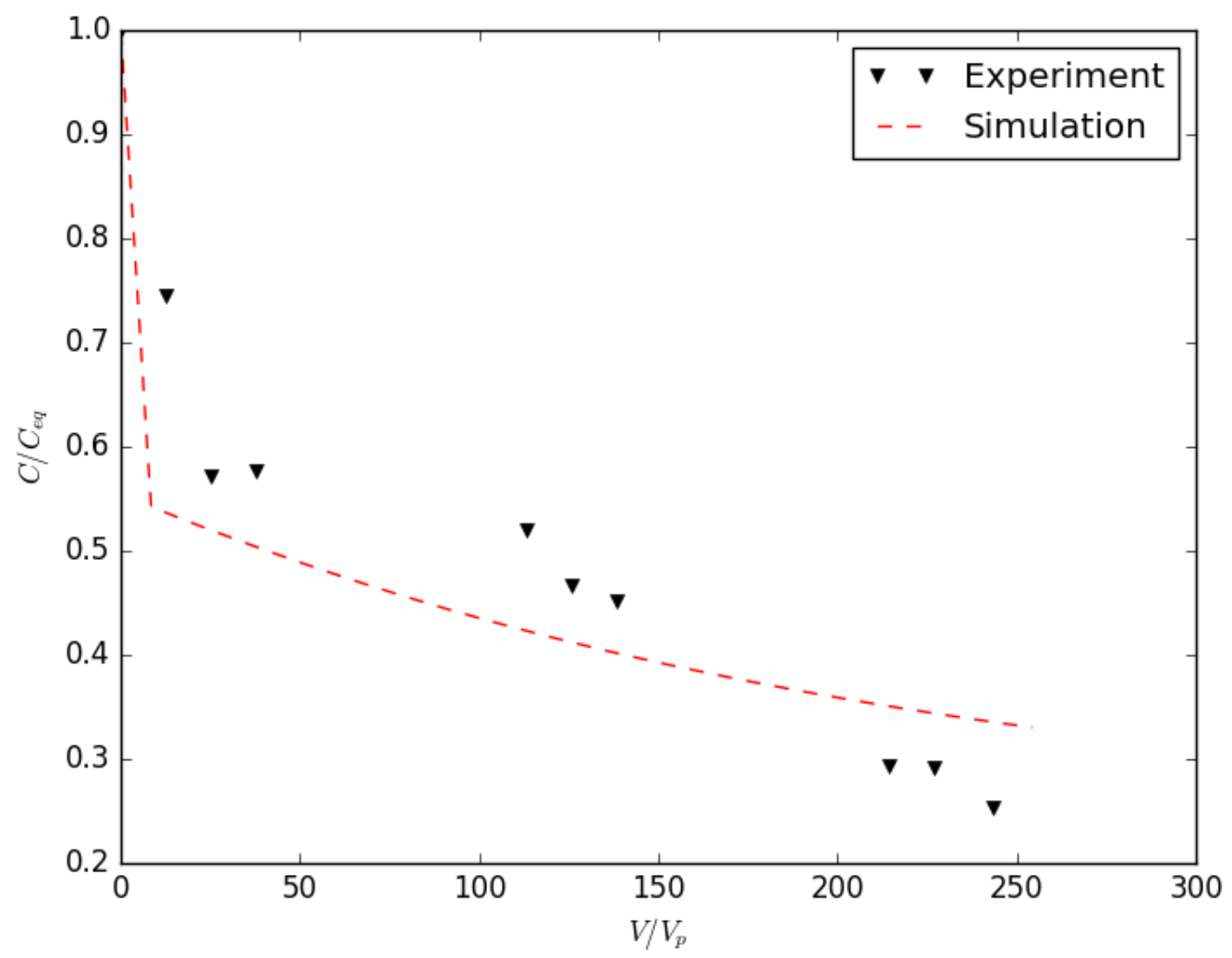

Figure 17: Normalized effluent concentration $\left(C / C_{e q}\right)$ versus pore volumes of water injected $\left(V / V_{p}\right)$ for $P_{e}=22$ (comparison between experimental results and numerical simulations)

\section{Conclusion}

In the first part of this work, we described the development of an experimental set-up using a micromodel to study the dissolution of pure organic phase in porous medium. To follow the dissolution of residual contaminant, two analytical approaches were considered and used: direct monitoring by image analysis and gas chromatography analysis. These two methods were assessed from mass balance calculation. Experiments were conducted at two flow rates and the resulting breakthrough curves of dissolved toluene and changes in NAPL saturation within the flowcell were measured. Results clearly demonstrated the influence of hydrodynamic conditions on NAPL dissolution and the increase of non-equilibrium phenomenon with the Péclet number.

In a second step, these experiments were used to validate the two-phase non-equilibrium theoretical model developped by (Quintard and Whitaker, 1994). The innovative approach proposed in this study is based on the calculation of the effective parameters (mass transfer coefficient, longitudinal dispersivity) over realistic pore-scale geometries derived from images of the porous medium. A significant advantage of our experimental set-up, indeed, is 
to provide a direct monitoring of NAPL dissolution with time that can be processed for obtaining 2D realistic configurations. Comparison of $1 \mathrm{D}$ macroscopic simulations with the experimental breakthrough curves indicates that the proposed model is able to describe with satisfactory accuracy the dissolution of NAPL in porous media and support a posteriori the calculation of effective coefficients. The differences between curves could be assigned to NAPL remobilization during the experiments and problems associated with the design of the micromodel, in particular with the reservoirs (presence of dead zones) that may induce a more or less significant error on the chromatographic measurements. As a conclusion, this study highlights the crucial need of having a fair recovery of pore-scale characteristic lengths to properly determine the changes with time in mass transfer mechanisms when using upscaling methods.

\section{Acknowledgements}

This work was partially supported by the French National Research Agency (ANR) through the MOBIOPOR project, with the reference ANR-10-BLAN-0908 and was performed as part of the French Scientific Interest Group-Industrial Wasteland (GISFI) program. This project has also received partial funding from the European Union's Horizon 2020 research and innovation program through the PROTINUS project under Grant Agreement No. 645717.

\section{References}

Abriola, L., 1989. Modeling multiphase migration of organic chemicals in groundwater systems- a review and assessment. Environmental Health Perspectives 83, 143-148.

Agaoglu, B., Scheytt, T., Copty, N., 2016. Impact of napl architecture on interphase mass transfer: A pore network study. Advances in Water Resources 95, 138-151.

Ahmadi, A., Aigueperse, A., Quintard, M., 2001. Calculation of the effective properties describing active dispersion in porous media: from simple to complex unit cells. Advances in Water Ressources 24, 423-431.

Al-Raoush, R., 2009. Impact of wettability on pore-scale characteristics of residual nonaqueous phase liquids. Environmental Science and Technology 43, 4796-4801.

Al-Raoush, R., 2014. Experimental investigation of the influence of grain geometry on residual napl using synchrotron microtomography. Journal of Contaminant Hydrology 159, 1-10.

Armstrong, R., Berg, S., 2013. Interfacial velocities and capillary pressure gradients during haines jumps. Physical Review E 88, 1-9.

Armstrong, R., McClure, J., Berrill, M., Rcker, M., Schlter, S., Berg, S., 2016. Beyond darcy's law: The role of phase topology and ganglion dynamics for two-fluid flow. Physical Review E 94, 043113.

Atteia, O., Jousse, F., Cohen, G., Hóhener, P., 2017. Comparison of residual napl source removal techniques in 3d metric scale experiments. Journal of Contaminant Hydrology 202, 23-32.

Bahar, T., Golfier, F., Oltéan, C., Benioug, M., 2016. An upscaled model for bio-enhanced napl dissolution in porous media. Transport in Porous Media 113, 653-693.

Borden, R., Kao, C.-M., 1992. Evaluation of groundwater extraction for remediation of petroleumcontaminated aquifers. Water Environment Research 64, 28-36.

Chomsurin, C., Werth, C., 2003. Analysis of pore-scale nonaqueous phase liquid dissolution in etched silicon pore networks. Water Resources Research 39, 1265.

Conrad, S., Wilson, J., Mason, W., Peplinski, W., 1992. Visualisation of residual organic liquid trapped in aquifers. Water Resources research 28, 467-478. 
Corapcioglu, M., Baehr, A., 1987. A compositional multiphase model for groundwater contamination by petroleum 1: Theroretical considerations. Water Resources Research 23, 191-200.

Corapcioglu, M., Yoon, S., Chowdhury, S., 2009a. Pore-Scale Analysis of NAPL Blob Dissolution and Mobilization in Porous Media. Transport in Porous Media 79, 419-442.

Corapcioglu, M., Yoon, S., Chowdhury, S., 2009b. Pore-scale analysis of napl blob dissolution and mobilization in porous media. Transport in Porous Media 79, 419-442.

Culligan, K., Wildenschild, D., Christensen, B., Gray, W., Rivers, M., 2006. Pore-scale characteristics of multiphase flow in porous media: A comparison of air-water and oil-water experiments. Advances in Water Resources 29, 227-238.

Cushman, H., Moroni, M., 2001. Statistical mechanics with three-dimensional particle tracking velocimetry experiments in the study of anomalous dispersion. i. theory. Phys. Fluids 13, 75-80.

Garing, C., de Chalendar, J., Voltolini, M., Ajo-Franklin, J., Benson, S., 2017. Pore-scale capillary pressure analysis using multi-scale x-ray micromotography. Advances in Water Resources 104, 223-241.

Ghosh, J., Tick, G., 2013. A pore scale investigation of crude oil distribution and removal from homogeneous porous media during surfactant-induced remediation. Journal of Contaminant Hydrology 155, 20 ?30.

Golfier, F., Quintard, M., Whitaker, S., 2002. Heat and mass transfer in tubes: An analysis using the method of volume averaging. Journal of Porous Media 5, 169-185.

Goyeau, B., Benihaddadene, T., Gobin, D., Quintard, M., 1999. Numerical calculation of the permeability in a dendritic mushy zone. Metall.and Mater.Trans 30B, 613-622.

Gray, W., Leijnse, A., Kolar, R., Blain, C., 1993. Mathematical tools for changing spatial scales in the analysis of physical systems. CRC Press: Boca Raton.

Hunt, J., Sitar, N., Udell, K., 1988. Nonaqueous phase liquid transport and cleanup: 1. analysis of mechanisms. Water Resources Research 24, 1247-1258.

Javanbakht, G., Arshadi, M., Qin, T., Goual, L., 2017. Micro-scale displacement of napl by surfactant and microemulsion in heterogeneous porous media. Advances in Water Resources 105, 173-187.

Javanbakht, G., Goual, L., 2016. Mobilization and micellar solubilization of napl contaminants in aquifer rocks. Journal of Contaminant Hydrology 185-186, 61-73.

Jeong, S.-W., Corapcioglu, M., Roosevelt, S., 2000. Micromodel study of surfactant foam remediation of residual trichloroethylene. Environ. Sci. Technol 34, 3456-3461.

Jia, C., Shing, K., Yortsos, Y., 1999. Visualization and simulation of non-aqueous phase liquids solubilization in pore networks. Journal of Contaminant Hydrology 25, 363-387.

Kashuk, S., Mercurio, S., Iskander, M., 2014. Visualization of dyed napl concentration in transparent porous media using color space components. Journal of Contaminant Hydrology 162-163, 1-16.

Kokkinaki, A., O'Carroll, D., Werth, C., Sleep, B., 2013. An evaluation of sherwoodgilland models for napl dissolution and their relationship to soil properties. Journal of Contaminant Hydrology 155, 87-98.

Mainhagu, J., Brusseau, M., 2016. Estimating initial contaminant mass based on fitting mass-depletion functions to contaminant mass discharge data: Testing method efficacy with sve operations data. Journal of Contaminant Hydrology 192, 152-157.

Miller, C., Poirier-McNeill, M., Mayer, A., 1990. Dissolution of trapped nonaqueous phase liquids : Mass transfer characteristics. Water Resources Research 26, 2783-2796.

Miller, C., Poirier-Mcnell, M., 1990. Dissolution of trapperd nonaqueous phase liquids : Mass transfer characteristics. Water Resources Research 26, 2783-2796.

Mobile, M., Widdowson, M., Stewart, L., Nyman, J., Deeb, R., Kavanaugh, M., Mercer, J., Gallagher, D., 2016. In-situ determination of field-scale napl mass transfer coefficients: Performance, simulation and analysis. Journal of Contaminant Hydrology 187, 31-46.

Nambi, I., Powers, S., 2003. Mass transfer correlations for nonaqueous phase liquid dissolution from regions with high initial saturations. Water Resources Research 39, 10-30.

National Research Council (NRC), 2005. Contaminants in the Subsurface: Source Zone Assessment and Remediation. National Academies Press.

Padgett, M., Tick, G., Carroll, K., Burke, W., 2017. Chemical structure influence on napl mixture nonideality evolution, rate-limited dissolution, and contaminant mass flux. Journal of Contaminant Hydrology 198, 
$11-23$.

Powers, S., Abriola, L., Weber, W., 1992. An experimental investigation of nonaqueous phase liquid dissolution in saturated subsurface systems : Steady state mass transfer rates. Water Resources Research 28, 2691-2705.

Powers, S., Abriola, L., Weber, W., 1994. An experimental investigation of nonaqueous phase liquid dissolution in saturated subsurface systems: Transient mass transfer rates. Water Ressources Research 30, $321-332$.

Quintard, M., Whitaker, S., 1994. Convection, dispersion, and interfacial transport of contaminants: Homogenous porous media. Advances in Water Ressources 17, 221-239.

Quintard, M., Whitaker, S., 1999. Dissolution of an immobile phase during flow in porous media. Ind. Eng. Chem. Res 38, 833-844.

Radilla, G., Quintard, M., Bertin, H., 1997. NAPL dissolution in saturated porous media: model experiments and pore scale interpretation. In: Sixth Symposium on multiphase transport in porous media. Vol. FED 244. pp. 423-429.

Radilla, G., Quintard, M., Bertin, H., 1998. Theoretical study and experimental validation of transport coefficients for hydrocarbon pollutants in aquifers. In: Recent Advances in Problems of Flow and Transport in Porous Media, ? Edition. Lewis publishers, pp. 143-152.

Rasband, W., 1997-2016. Image J. U.S. National Institutes of Health, Bethesda, Maryland, USA, https://imagej.nih.gov/ij/.

Saba, T., Illangasekare, T., 2000. Effect of groundwater flow dimensionality on mass transfer from entrapped nonaqueous phase liquid contaminants. Water Resources Research 36, 971-979.

Sahloul, N., Ioannidis, M., Chatzis, I., 2002. Dissolution of residual non-aqueous phase liquids in porous media: pore-scale mechanisms and mass transfer rates. Advances in Water Resources 25, 33-49.

Schubert, M., Paschke, A., Lau, S., Geyer, W., Knöller, K., 2007. Radon as a naturally occurring tracer for the assessment of residual napl contamination of aquifers. Environmental Pollution 145, 920-927. URL https://doi.org/10.1016/j.envpol.2006.04.029

Seagren, E., Rittmann, B., Valocchi, A., 1999. A critical evaluation of the local-equilibrium assumption in modeling napl-pool dissolution. Journal of Contaminant Hydrology 39, 109-135.

Wehrer, M., Mai, J., Attinger, S., Totsche, K., 2013. Kinetic control of contaminant release from napls information potential of concentration time profiles. Environmental Pollution 179, 301-314.

Whitaker, S., 1999. The method of volume averaging. Dordrecht: Kluwer Academic Publishers.

Yra, A., Bertin, H., Ahmadi, A., 2006. étude expérimentale de la dispersion active d'un polluant hydrocarboné en milieu poreux hétérogène. C. R. Mecanique 334, 58-67.

Zhao, W., Ioannidis, M., 2003. Pore network simulation of the dissolution of a single component wetting nonaqueous phase liquid. Water Resources Research 39, 1291.

Zhao, W., Ioannidis, M., 2007. Effect of napl film stability on the dissolution of residual wetting napl in porous media: A pore-scale modeling study. Advances in Water Resources 30, 171-181.

\section{AppendixA. Upscaling of the mass transport equations}

We begin our analysis by applying the spatial and temporal averaging theorems, that can be expressed as (Whitaker, 1999):

$$
\begin{aligned}
&\left\langle\nabla c_{A \beta}\right\rangle=\nabla\left\langle c_{A \beta}\right\rangle+ \frac{1}{V} \int_{A_{\beta \gamma}} \mathbf{n}_{\beta \gamma} c_{A \beta} d A+\frac{1}{V} \int_{A_{\beta \sigma}} \mathbf{n}_{\beta \sigma} c_{A \beta} d A \\
&\left\langle\nabla \cdot\left(\mathbf{v}_{A \beta} c_{A \beta}\right)\right\rangle= \nabla \cdot\left\langle\mathbf{v}_{A \beta} c_{A \beta}\right\rangle+\frac{1}{V} \int_{A_{\beta \gamma}} \mathbf{n}_{\beta \gamma} \cdot\left(\mathbf{v}_{A \beta} c_{A \beta}\right) d A \\
&+\frac{1}{V} \int_{A_{\beta \sigma}} \mathbf{n}_{\beta \sigma} \cdot\left(\mathbf{v}_{A \beta} c_{A \beta}\right) d A \\
& 36
\end{aligned}
$$




$$
\left\langle\frac{\partial c_{A \beta}}{\partial t}\right\rangle=\frac{\partial\left\langle c_{A \beta}\right\rangle}{\partial t}-\frac{1}{V} \int_{A_{\beta \gamma}} \mathbf{n}_{\beta \gamma} \cdot c_{A \beta} \mathbf{w}_{\beta \gamma} d A-\frac{1}{V} \int_{A_{\beta \sigma}} \mathbf{n}_{\beta \sigma} \cdot c_{A \beta} \mathbf{w}_{\beta \sigma} d A
$$

The volume averaging theorems have been used for obtaining the averaged transport equation in each phase:

$\beta$-phase

$$
\begin{aligned}
& \underbrace{\frac{\partial\left(\epsilon_{\beta}\left\langle c_{A \beta}\right\rangle^{\beta}\right)}{\partial t}}_{\text {Accumulation }}+\underbrace{\nabla \cdot\left\langle\mathbf{v}_{A \beta} c_{A \beta}\right\rangle}_{\text {Advection }}+\underbrace{\frac{1}{V} \int_{A_{\beta \gamma}} \mathbf{n}_{\beta \gamma} \cdot c_{A \beta}\left(\mathbf{v}_{A \beta}-\mathbf{w}_{\beta \gamma}\right) d A}_{\text {Interfacial flux }} \\
& +\underbrace{\frac{1}{V} \int_{A_{\beta \sigma}} \mathbf{n}_{\beta \sigma} \cdot c_{A \beta}\left(\mathbf{v}_{A \beta}-\mathbf{w}_{\beta \sigma}\right) d A}_{\text {Interfacial flux }}=0
\end{aligned}
$$

$\gamma$-phase

$$
\underbrace{\frac{\partial\left\langle\rho_{\gamma}\right\rangle}{\partial t}}_{\text {Accumulation }}+\underbrace{\frac{1}{V} \int_{A_{\beta \gamma}} \rho_{\gamma} \mathbf{n}_{\beta \gamma} \cdot \mathbf{w}_{\beta \gamma} d A}_{\text {Interfacial flux }}=0
$$

Taking into account the relationships expressing the condition of a dilute solution of species $A$ (additional details are available in Quintard and Whitaker (1999)),

$$
c_{A \beta} \mathbf{v}_{A \beta}=c_{A \beta} \mathbf{v}_{\beta}-D_{A \beta} \nabla c_{A \beta}
$$

the superficial average equation, Eq. A.4, in the $\beta$-phase becomes:

$\beta$-phase

$$
\begin{aligned}
& \underbrace{\frac{\partial\left(\epsilon_{\beta}\left\langle c_{A \beta}\right\rangle^{\beta}\right)}{\partial t}}_{\text {Accumulation }}+\underbrace{\nabla \cdot\left\langle\mathbf{v}_{\beta} c_{A \beta}\right\rangle}_{\text {Advection }}=-\underbrace{\frac{1}{V} \int_{A_{\beta \gamma}} \mathbf{n}_{\beta \gamma} \cdot\left(c_{A \beta}\left(\mathbf{v}_{\beta}-\mathbf{w}_{\beta \gamma}\right)-D_{A \beta} \nabla c_{A \beta}\right) d A}_{\text {Interfacial flux }} \\
& +\underbrace{\nabla \cdot\left\langle D_{A \beta} \nabla c_{A \beta}\right\rangle}_{\text {Dispersion }}-\underbrace{\frac{1}{V} \int_{A_{\beta \sigma}} \mathbf{n}_{\beta \sigma} \cdot\left(c_{A \beta}\left(\mathbf{v}_{\beta}-\mathbf{w}_{\beta \sigma}\right)-D_{A \beta} \nabla c_{A \beta}\right) d A}_{\text {Interfacial flux }}
\end{aligned}
$$

Finally the unclosed form of the averaged equation in the $\beta$-phase can be expressed as (the complete development for averaged equations is available in Quintard and Whitaker (1994)): 


\section{$\beta$-phase}

$$
\begin{aligned}
& \underbrace{\frac{\partial\left\langle c_{A \beta}\right\rangle^{\beta}}{\partial t}}_{\text {Accumulation }}+\underbrace{\left\langle\mathbf{v}_{\beta}\right\rangle^{\beta} \cdot \nabla\left\langle c_{A \beta}\right\rangle^{\beta}}_{\text {Advection }}+\underbrace{\epsilon_{\beta}^{-1} \nabla \cdot\left\langle\tilde{\mathbf{v}}_{\beta} \tilde{c}_{A \beta}\right\rangle}_{\text {Dispersive transport }}=\underbrace{\nabla \cdot\left(D_{A \beta} \nabla\left\langle c_{A \beta}\right\rangle^{\beta}\right)}_{\text {Dispersion }} \\
& +\underbrace{\epsilon_{\beta}^{-1} \nabla \cdot\left[D_{A \beta}\left(\frac{1}{V} \int_{A_{\beta \gamma}} \mathbf{n}_{\beta \gamma} \tilde{c}_{A \beta} d A+\frac{1}{V} \int_{A_{\beta \sigma}} \mathbf{n}_{\beta \sigma} \tilde{c}_{A \beta} d A\right)\right.}_{\text {Dispersion }}-\underbrace{\frac{\epsilon_{\beta}^{-1}}{V} \int_{A_{\beta \gamma}} \mathbf{n}_{\beta \gamma} \cdot \tilde{c}_{A \beta}\left(\mathbf{v}_{\beta}-\mathbf{w}_{\beta \gamma}\right) d A}_{\text {Interfacial flux }} \\
& -\underbrace{\frac{\epsilon_{\beta}^{-1}}{V} \int_{A_{\beta \sigma}} \mathbf{n}_{\beta \sigma} \cdot \tilde{c}_{A \beta}\left(\mathbf{v}_{\beta}-\mathbf{w}_{\beta \sigma}\right) d A+\frac{\epsilon_{\beta}^{-1}}{V} \int_{A_{\beta \gamma}} \mathbf{n}_{\beta \gamma} \cdot D_{A \beta} \nabla \tilde{c}_{A \beta} d A}_{\text {Interfacial flux }} \\
& +\underbrace{\frac{\epsilon_{\beta}^{-1}}{V} \int_{\mathbf{n}_{\beta \sigma} \cdot D_{A \beta} \nabla \tilde{c}_{A \beta} d A}}_{A_{\beta \sigma}}
\end{aligned}
$$

For a binary system and under the dilute solution approximation Quintard and Whitaker, 1999), the velocity of interface $A_{\beta \gamma}$ can be expressed as,

$$
\mathbf{w}_{\beta \gamma} \cdot \mathbf{n}_{\beta \gamma}=\frac{1}{\rho_{\gamma}} \mathbf{n}_{\beta \gamma} \cdot D_{A \beta} \nabla c_{A \beta} \quad \text { at } A_{\beta \gamma}
$$

Finally, substituting the last equation, Eq. A.9], into the averaged equation, Eq A.5, we derived the unclosed form of macroscopic equation in the $\gamma$-phase:

$$
\underbrace{\rho_{\gamma} \frac{\partial \epsilon_{\gamma}}{\partial t}}_{\text {Accumulation }}+\underbrace{\frac{1}{V} \int_{A_{\beta \gamma}} \mathbf{n}_{\beta \gamma} \cdot D_{A \beta} \nabla\left\langle c_{A \beta}\right\rangle^{\beta} d A+\frac{1}{V} \int_{A_{\beta \gamma}} \mathbf{n}_{\beta \gamma} \cdot D_{A \beta} \nabla \tilde{c}_{A \beta} d A}_{\text {Interfacial flux }}=0
$$

\section{AppendixA.1. Closure}

Firstly, we recall our original differential equation defined in the $\beta$ - phase at the pore scale,

$$
\frac{\partial\left(c_{A \beta}\right)}{\partial t}+\nabla \cdot\left(\mathbf{v}_{\beta} c_{A \beta}\right)=\nabla \cdot\left(D_{A \beta} \nabla c_{A \beta}\right)
$$

By subtracting Eq.A.8 from Eq.A.11, we obtain the set of equations governing the con- 
centration deviations:

$$
\begin{aligned}
& \mathbf{v}_{\beta} \cdot \nabla \tilde{c}_{A \beta}+\tilde{\mathbf{v}}_{\beta} \cdot \nabla\left\langle c_{A \beta}\right\rangle^{\beta}=\nabla \cdot\left(D_{A \beta} \nabla \tilde{c}_{A \beta}\right)+\frac{\epsilon_{\beta}^{-1}}{V} \int_{A_{\beta \gamma}} \mathbf{n}_{\beta \gamma} \cdot \tilde{c}_{A \beta}\left(\mathbf{v}_{\beta}-\mathbf{w}_{\beta \gamma}\right) d A \\
& +\frac{\epsilon_{\beta}^{-1}}{V} \int_{A_{\beta \sigma}} \mathbf{n}_{\beta \sigma} \cdot \tilde{c}_{A \beta}\left(\mathbf{v}_{\beta}-\mathbf{w}_{\beta \sigma}\right) d A-\frac{\epsilon_{\beta}^{-1}}{V} \int_{A_{\beta \gamma}} \mathbf{n}_{\beta \gamma} \cdot D_{A \beta} \nabla \tilde{c}_{A \beta} d A \\
& -\frac{\epsilon_{\beta}^{-1}}{V} \int_{A_{\beta \sigma}} \mathbf{n}_{\beta \sigma} \cdot D_{A \beta} \nabla \tilde{c}_{A \beta} d A \\
& \text { B.C.1 }-\underbrace{\mathbf{n}_{\beta \sigma} \cdot D_{A \beta} \nabla\left\langle c_{A \beta}\right\rangle^{\beta}}_{\text {Source }}=\mathbf{n}_{\beta \sigma} \cdot D_{A \beta} \nabla \tilde{c}_{A \beta} \quad \text { at } A_{\beta \sigma} \\
& \text { B.C.2 } \quad \tilde{c}_{A \beta}=\underbrace{c_{A \beta}^{e q}-\left\langle c_{A \beta}\right\rangle^{\beta}}_{\text {Source }} \quad \text { at } A_{\beta \gamma} \\
& \text { B.C.3 (Periodicity) } \quad \tilde{c}_{A \beta}\left(\mathbf{r}+l_{i}\right)=\tilde{c}_{A \beta}(\mathbf{r}), \quad i=1,2,3 \\
& \text { B.C.4 }\left\langle\tilde{c}_{A \beta}\right\rangle^{\beta}=0
\end{aligned}
$$

Following the developments in the literature (e.g., Quintard and Whitaker (1999)), we identify two non-homogeneous terms, $\nabla\left\langle c_{A \beta}\right\rangle^{\beta}$ and $\left(c_{A \beta}^{e q}-\left\langle c_{A \beta}\right\rangle^{\beta}\right)$, that act as source terms for the deviation fields. We use these terms to formulate the closure problem solution, i.e., a solution of the form:

$$
\tilde{c}_{A \beta}=\mathbf{b}_{\beta} \cdot \nabla\left\langle c_{A \beta}\right\rangle^{\beta}+s_{\beta}\left(c_{A \beta}^{e q}-\left\langle c_{A \beta}\right\rangle^{\beta}\right)
$$

where the closure variables $\mathbf{b}_{\beta}$ and $s_{\beta}$ satisfy the closure problems obtained by substituting Eqs. A.17 into the deviations equations. The two closure problems are specified below in their dimensionless form:

\section{Closure problems (dimensionless form)}

\section{Problem I}

$$
\begin{aligned}
& \beta \text {-phase: } \quad P e_{A}\left(\tilde{\mathbf{v}}_{\beta}^{\prime}+\mathbf{v}_{\beta}^{\prime} \cdot \nabla \mathbf{b}_{\beta}^{\prime}\right)=\nabla^{2} \mathbf{b}_{\beta}^{\prime}-\epsilon_{\beta}^{-1} \mathbf{U}_{\beta}^{\prime} \\
& \text { B.C.1 }-\mathbf{n}_{\beta \sigma} \cdot \nabla \mathbf{b}_{\beta}^{\prime}=\mathbf{n}_{\beta \sigma} \quad \text { at } A_{\beta \sigma} \\
& \text { B.C.2 } \quad \mathbf{b}_{\beta}^{\prime}=0 \quad \text { at } A_{\beta \gamma} \\
& \text { B.C.3 (Periodicity) } \quad \mathbf{b}_{\beta}^{\prime}\left(\mathbf{r}+l_{i}\right)=\mathbf{b}_{\beta}^{\prime}(\mathbf{r}), i=1,2,3 \quad i=1,2,3 \\
& \text { B.C.4 }\left\langle\mathbf{b}_{\beta}^{\prime}\right\rangle^{\beta}=0 \\
& \mathbf{U}_{\beta}^{\prime}=\frac{1}{V} \int_{A_{\beta \gamma}} \mathbf{n}_{\beta \gamma} \cdot \nabla \mathbf{b}_{\beta}^{\prime} d A+\frac{1}{V} \int_{A_{\beta \sigma}} \mathbf{n}_{\beta \sigma} \cdot \nabla \mathbf{b}_{\beta}^{\prime} d A
\end{aligned}
$$




\section{Problem II}

$$
\begin{aligned}
\beta \text {-phase: } \quad P e_{A} \mathbf{v}_{\beta}^{\prime} \cdot \nabla s_{\beta}^{\prime}=\nabla^{2} s_{\beta}^{\prime}-\epsilon_{\beta}^{-1} \mathbf{S}_{\beta}^{\prime} \\
\text { B.C.1 } \quad-\mathbf{n}_{\beta \sigma} \cdot \nabla s_{\beta}^{\prime}=0 \quad \text { at } A_{\beta \sigma} \\
\text { B.C.2 } \quad s_{\beta}^{\prime}=1 \quad \text { at } A_{\beta \gamma} \\
\text { B.C.3 (Periodicity) } \quad s_{\beta}^{\prime}\left(\mathbf{r}+l_{i}\right)=s_{\beta}^{\prime}(r), i=1,2,3 \quad i=1,2,3 \\
\text { B.C.4 } \quad\left\langle s_{\beta}^{\prime}\right\rangle^{\beta}=0 \\
\mathbf{S}_{\beta}^{\prime}=\frac{1}{V} \int_{A_{\beta \gamma}} \mathbf{n}_{\beta \gamma} \cdot \nabla s_{\beta}^{\prime} d A+\frac{1}{V} \int_{A_{\beta \sigma}} \mathbf{n}_{\beta \sigma} \cdot \nabla s_{\beta}^{\prime} d A
\end{aligned}
$$

where the dimensionless variables and the parameters have been defined by

$$
\tilde{\mathbf{v}}_{\beta}^{\prime}=\frac{\tilde{\mathbf{v}}_{\beta}}{\left\|\mathbf{v}_{\beta}\right\|} \quad \mathbf{v}_{\beta}^{\prime}=\frac{\mathbf{v}_{\beta}}{\left\|\mathbf{v}_{\beta}\right\|} \quad P e_{A}=\frac{\left\|\mathbf{v}_{\beta}\right\| l_{\beta}}{D_{A \beta}}
$$

AppendixA.2. Closed Form of the Macroscopic Equations

Now we introduce the closure solution, Eq. A.17, into the unclosed averaged equation. The following closed form of the mass transport equations is obtained:

$$
\begin{gathered}
\frac{\partial \epsilon_{\beta}\left\langle c_{A \beta}\right\rangle^{\beta}}{\partial t}+\epsilon_{\beta}\left\langle\mathbf{v}_{\beta}\right\rangle^{\beta} \cdot \nabla\left\langle c_{A \beta}\right\rangle^{\beta}-\mathbf{d}_{\beta \omega} \cdot \nabla\left\langle c_{A \beta}\right\rangle^{\beta}-\mathbf{U}_{\beta \omega} \cdot \nabla\left\langle c_{A \beta}\right\rangle^{\beta}= \\
\nabla \cdot\left(\mathbf{D}_{\beta \omega}^{*} \cdot \nabla\left\langle c_{A \beta}\right\rangle^{\beta}\right)+\alpha_{A}^{*}\left(c_{A \beta}^{e q}-\left\langle c_{A \beta}\right\rangle^{\beta}\right) \\
\rho_{\gamma} \frac{\partial \epsilon_{\gamma}}{\partial t}=-\alpha_{A}^{*}\left(c_{A \beta}^{e q}-\left\langle c_{A \beta}\right\rangle^{\beta}\right)
\end{gathered}
$$

